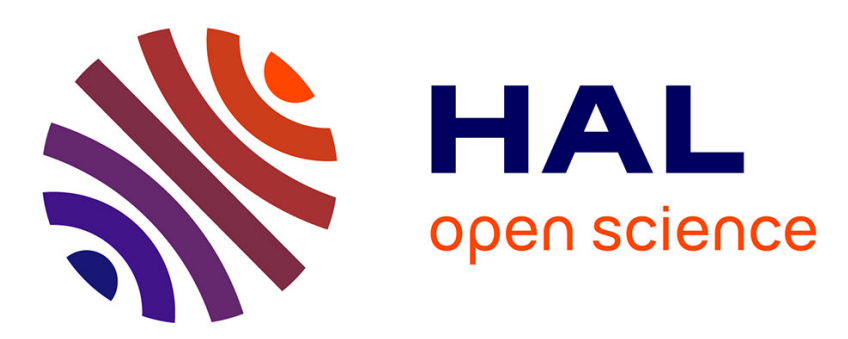

\title{
La publicité comparative, ou comment se faire justice à soi-même en passant par le droit
}

Franck Cochoy, Roland Canu

\section{To cite this version:}

Franck Cochoy, Roland Canu. La publicité comparative, ou comment se faire justice à soi-même en passant par le droit. Revue française de sociologie, 2006, 47 (1), pp.81-115. hal-00186564

\section{HAL Id: hal-00186564 https://hal.science/hal-00186564}

Submitted on 12 Nov 2007

HAL is a multi-disciplinary open access archive for the deposit and dissemination of scientific research documents, whether they are published or not. The documents may come from teaching and research institutions in France or abroad, or from public or private research centers.
L'archive ouverte pluridisciplinaire HAL, est destinée au dépôt et à la diffusion de documents scientifiques de niveau recherche, publiés ou non, émanant des établissements d'enseignement et de recherche français ou étrangers, des laboratoires publics ou privés. 


\title{
«La publicité comparative, ou comment se faire justice à soi-même en passant par le droit »
}

\author{
Franck Cochoy et Roland Canu
}

\begin{abstract}
Résumé. La publicité comparative convoque non seulement celui qui y recourt et le (ou les) concurrent(s) qu'il désigne, mais aussi le droit, qui sert de point d'appui et de cadre à leur confrontation. Ainsi, en permettant de « se faire justice à soi-même en passant par le droit », la publicité comparative met en jeu la redéfinition conjointe et constante d'une pratique économique et de h règle censée l'encadrer. Cette redéfinition est le fruit de trois hésitations successives. La première, historique, est celle des régulateurs qui incorporent peu à peu la notion de publicité comparative dans le droit économique tout en restreignant considérablement sa pratique. La seconde, stratégique, est celle des entreprises qui, cherchant à jauger l'opportunité de s'engager dans la publicité comparative ou non, explorent au moins deux manières de jouer avec le droit : comparer leur produit à un concurrent fictif pour éviter le cadre légal, ou bien se situer au contraire résolument dans ce cadre comme si les acteurs cherchaient à faire en sorte que $\mathrm{e}$ droit fonctionne comme un label malgré lui. La troisième hésitation, sociale, est celle des acteurs (plaignants, avocats, juge), dont la tâche consiste à accommoder les ambiguïtés des deux autres : grâce à l'examen d'un cas particulier de jurisprudence, l'article montre à quel point la publicité comparative amène le juge à composer pragmatiquement avec les règles de droit mais aussi avec les données de l'économie. L'usage de la publicité comparative circonscrit ainsi un espace d'expérimentation collective dans lequel les acteurs économiques parviennent à éprouver, renforcer ou redéfinir continûment les normes et les pratiques de l'échange marchand.
\end{abstract}

Correspondance: F. Cochoy, CERTOP, UMR CNRS 5044, Maison de la Recherche, 5, allées Antonio Machado, 31058, Toulouse CEDEX. E-mail : cochoy@univ-tlse2.fr. Tél. 0562 160609 
La publicité occupe une place paradoxale dans les travaux de sciences sociales. D'un côté, c'est l'un des rares outils commerciaux qui ait réussi à mobiliser depuis longtemps l'attention des sociologues, que ces derniers s'intéressent à la publicité comme instrument de manipulation marchande (Baudrillard, 1970), comme «système magique » associant la consommation aux désirs humains (Williams, 1990), comme agent de contrôle social (Ewen, 1983), ou encore comme instance productrice d'imaginaire (Sauvageot, 1987) ${ }^{1}$. Mais d'un autre côté, les sciences sociales ne retiennent généralement qu'un seul aspect de la publicité : celui qui manipule et non celui qui informe, celui qui mobilise des images et des représentations, et non celui qui indexe les objets du monde commercial sous la forme d'une représentation plus ou moins sélective et imagée. Ce faisant, les analystes laissent de côté les enseignements de l'histoire qui nous montrent combien la publicité trouve ses racines dans l'information économique avec les mercuriales (Cochran, 1977) et entretient depuis toujours des relations certes complexes mais néanmoins tangibles avec ses sous-jacents marchands, tant aux États-Unis (Marchand, 1986) qu'en France (Chessel, 1998). Ici nous voudrions, en prenant appui sur le cas de la publicité comparative, revisiter ces tensions inhérentes au média publicitaire qu'une perspective strictement critique tend à oublier. Plus encore, nous entendons montrer que la publicité comparative met en jeu non seulement les ambiguïtés et les ressorts historiques du média auquel elle se rattache, mais aussi et surtout une certaine reconfiguration du rapport marchand, qui se donne à lire au carrefour de l'économie et du droit.

En effet, pour les acteurs du marché comme pour les théoriciens de l'économie économistes mais aussi sociologues —, la publicité comparative pose deux problèmes : celui de la publicité et celui de la comparaison! Le problème de la publicité est celui dont nous sommes partis, et se joue autour de deux tendances contradictoires qui structurent, depuis les travaux d'Alfred Marshall, la littérature économique sur le sujet (Doyle, 1968). D'un côté, toute publicité contribue à la manipulation de l'information au profit de l'acteur ou du groupe d'acteurs qui la met en œuvre, dans la mesure où la publicité est évidemment d'abord au service de celui qui la paye. De longue date, les risques (macro) économiques associés à cette dimension «combative » (Marshall, 1922) de l'action publicitaire sont discutés par bon nombre de théoriciens (Kaldor, 1950 ; Telser, 1964 ; Comanor \& Wilson, 1979). Mais d'un autre côté et a contrario, la publicité contribue aussi à performer l'hypothèse d'information parfaite des agents en situation de concurrence, puisque ce média est l'un des principaux vecteurs d'information économique à destination des marchés (Stigler, 1961 ; Nelson, 1975 ; Coase, 1977). Le problème de la comparaison est celui qui naît de la mise en équivalence de biens génériques dont la publicité comparative entend pourtant signaler les différences. Pour l'économie théorique standard, le choix du consommateur est censé porter sur des biens radicalement différents (par exemple du vin et du pain chez Pareto), chacun des biens

1. Ces vingt dernières années le désintérêt relatif des sociologues pour les dispositifs de médiation marchande a fort heureusement tendu à s'effacer, avec la multiplication de travaux consacrés non seulement à la publicité (Hennion \& Méadel, 1988) mais bientôt au design (Hennion \& Dubuisson, 1996 ; Vervaeke, 2004), au marketing (Cochoy, 1999), au packaging (Cochoy, 2002a), aux guides d'achat (Karpik, 2000 ; Mallard, 2000a) et au merchandising (Barrey, 2004 ; Grandclément, 2004 ; Du Gay, 2004), ou bien encore à des dispositifs plus larges, comme les normes (Mallard, 2000b; Cochoy, 2000 ; Borraz, 2004) et autres outils de calcul (Callon \& Muniesa, 2004), etc. 
constitutifs du choix étant considéré comme parfaitement homogène (dans le modèle canonique du choix du consommateur, toutes les unités d'un même produit sont présumées identiques en prix et en qualité). Or la publicité comparative définit d'une part une situation de choix entre le même et le même, qui place le consommateur dans une hésitation analogue à celle de l'âne de Buridan (Cochoy, 2002a), et d'autre part — et paradoxalement — la même publicité introduit simultanément le soupçon de l'absence d'identité des biens qu'elle rapproche (la publicité comparative entend révéler une asymétrie d'information entre ces biens supposés «comparables », aux deux sens contradictoires de cet adjectif: identiques et différenciables). Notons immédiatement que l'économie théorique n'a attendu ni l'anthropologie des marchés ni même la publicité comparative pour investir cette double tension (que cette forme de publicité ne fait que mettre au jour et radicaliser) et pour tenter d'ajuster ses cadres d'analyse en conséquence. Depuis Chamberlin (1932), la théorie de la concurrence oligopolistique prend en compte l'existence d'une pluralité de marchés organisant chacun la différenciation des occurrences singulières d'une même catégorie de produits ; or, parmi les principaux éléments intervenant dans cette organisation, la publicité figure en bonne place, aux côtés des prix et de la nature des produits (ibid.). Plus tard, parallèlement à l'exploration très féconde des «économies de la qualité » (Akerlof, 1970 ; Lancaster, 1975 ; Spence, 1973) ${ }^{2}$, d'autres économistes ont examiné plus précisément les liens existant entre la publicité et la révélation des attributs qualitatifs des produits aux consommateurs (Nelson 1970 ; Nelson, 1974 ; Kihlstrom et Riordan, 1984).

La prise en charge progressive de cette double tension par la discipline économique semble donc indiquer que si la publicité comparative pose un problème, c'est moins un problème théorique - qui offrirait une nouvelle occasion au spécialiste de sociologie économique de reprendre à nouveaux frais sa querelle classique contre les « fictions de l'économie » — qu'une difficulté pragmatique a priori, celle que représente la nécessité du réglage, pour et par les acteurs de l'économie — juristes, managers, consommateurs — du délicat équilibre entre les deux bords de chacune des tensions que nous avons signalées. En d'autres termes, avec la publicité comparative, l'hésitation qui entoure le fonctionnement de l'économie n'est pas tant le problème des sciences sociales que celui des acteurs du marché ; partant, la tâche de la sociologie économique ne consiste plus à instruire la double critique de l'économie (comme savoir et comme discipline) mais à rendre compte des critiques endogènes à l'action économique $^{3}$. Dans le cas de la publicité comparative la critique est double, puisqu'elle convoque à la fois les plaignants et le droit, au terme d'un processus très particulier qui met en

2. Pour une revue de littérature, Cf. par exemple Tirole (1995).

3. On aura noté que ce changement de perspective consiste à mettre en œuvre, du côté de la sociologie économique, l'aggiornamento salutaire proposé par Luc Boltanski consistant à passer de la sociologie critique à une sociologie de la critique (Boltanski, 1990) — un mouvement aujourd'hui prolongé par la mise en débat de l'«académisme radical » (Lapeyronnie, 2004). Au lieu de réduire la publicité aux éléments qui méritent la dénonciation (Cf. supra), mieux vaut selon nous montrer que la dénonciation de la publicité est endogène au monde économique, afin de comprendre que les consommateurs ne sont pas les seules victimes des agissements publicitaires (la publicité comparative met aussi en question l'identité, voire l'existence des concurrents), et de mettre au jour le rôle crucial du droit dans la structuration de cet espace de tensions. 
jeu la redéfinition conjointe et constante d'une pratique économique et de la règle censée l'encadrer'.

Cette redéfinition est le fruit de trois hésitations successives et articulées l'une à l'autre. La première, historique, est celle des régulateurs, qui incorporent peu à peu la notion de publicité comparative dans le droit économique tout en restreignant considérablement sa pratique : nous serons ainsi conduits à examiner la façon dont le droit a construit/contraint la publicité comparative, en prenant soin d'inscrire cette histoire dans le cadre plus général des travaux qui se sont intéressés aux rapports entre l'économie et le droit. La seconde hésitation, stratégique, est celle des entreprises qui, cherchant à jauger l'opportunité de s'engager dans cette pratique ou non, explorent diverses manières de jouer avec le droit : par exemple, certaines optent pour le registre de la comparaison en se tenant toutefois soigneusement à l'écart du cadre légal qui régit la publicité comparative, d'autres au contraire choisissent de se situer résolument dans ce cadre... dans l'espoir de capter aussi, en retour, la légitimité associée à l'autorité juridique. La troisième hésitation, sociale, est celle des acteurs (plaignants, avocats, juge), dont la tâche consiste à composer avec les ambiguïtés des deux autres : grâce à l'examen d'un cas particulier de jurisprudence, nous verrons à quel point l'usage des publicités comparatives circonscrit un espace d'expérimentation collective dans lequel (ou grâce auquel ?) les acteurs économiques parviennent à éprouver, renforcer ou redéfinir continûment les normes et les pratiques de l'échange marchand ${ }^{5}$. En étudiant de près cet ensemble d'hésitations, nous espérons montrer que les activités sociales qui se déploient autour de la publicité comparative, loin de s'inscrire passivement dans le cadre du droit et/ou de répondre mécaniquement aux impératifs du monde économique, contribuent plutôt à redéfinir l'un et l'autre6 .

4. Notre démarche rejoint les travaux portant sur la façon dont les pratiques marchandes performent les catégories de l'économie et autres représentations du marché (Callon, 1998): les savoirs économiques, gestionnaires ou juridiques seront considérés comme performatifs chaque fois qu'ils définissent et mettent en forme simultanément les réalités marchandes.

5. La présente enquête, conformément au parti pris retenu ici qui consiste à étudier une question publique à partir des points de vue exprimés publiquement, s'appuie sur le recueil du corpus juridique traitant de la publicité comparative, sur l'exploitation des débats publics concernant ce média, tels qu'ils apparaissent dans la presse, notamment dans le Journal le Monde, mais aussi sur l'examen des campagnes et/ou de la jurisprudence concernant le cas exemplaire de Télé2 vs. France Telecom. Les jugements ayant opposé ces deux entreprises autour de la publicité comparative ont été recueillis et analysés ; l'intérêt de ce matériau est de rassembler les points de vue qui font sens pour les acteurs — plaignants représentés par leurs avocats, juges — et donc de donner un accès direct à la controverse et aux positions de chacun tels qu'ils ont été directement échangés et forgés, dans le cours de l'action. Nous rejoignons ici la perspective d'Alessandro Stanziani, pour qui «l'analyse des contentieux [judiciaires] permet de comprendre la manière dont les acteurs économiques et institutionnels perçoivent les règles et les mettent en pratique » $(2003 \mathrm{a} ;$ p. 64).

6. Nous remercions Sandrine Barrey et Martin Giraudeau pour leur lecture d'une version antérieure de ce texte, ainsi que les membres du comité de lecture de la revue, en particulier Denis Segrestin (qui a rassemblé leurs avis) ainsi qu'un autre expert anonyme, pour leur contribution très précise et constructive à l'amélioration de ce texte. Bien entendu les propos tenus ici n'engagent que les auteurs. 


\section{Des hésitations à entrouvrir la brèche : une histoire juridique de la publicité comparative}

L'histoire juridique de la publicité comparative en France, telle que nous proposons de la retracer brièvement dans cette première partie, engage d'abord la question des rapports entre droit et marché. Parce qu'il donne à voir un droit chargé d'assurer des pratiques concurrentielles conformes à certaines conceptions de l'économie mais aussi à des considérations propres à la justice, l'examen de la régulation de cette pratique s'inscrit dans une longue tradition de recherches portée actuellement par des courants très actifs, au premier rang desquels figurent notamment le Droit Économique (du côté des juristes) et l'Analyse Économique du Droit (du côté des économistes) 7 . Ces travaux, aussi composites soient-ils, avancent quelques idées fortes. Nous en retiendrons deux que la publicité comparative vient singulièrement éprouver. La première idée concerne le caractère consubstantiel au marché et notamment aux mécanismes concurrentiels - d'une «hétéro-régulation » (Champaud, 2002) dont les règles législatives feraient partie. Bon nombre de ces travaux aspirent en effet à se détacher d'une vision naturaliste de la concurrence, telle qu'elle est présentée par la théorie économique classique, pour considérer plutôt que les régulations, notamment juridiques, sont nécessaires au déroulement du jeu marchand. Cette idée se retrouve, formalisée de différentes façons, aussi bien du côté de certains versants de l'économie institutionnelle (Commons, 1925 ; Commons, 1931), que de la sociologie économique (Steiner, 1999; Trigilia, 2002) ${ }^{8}$, de l'histoire sociale des affaires (Fligstein, 1990), ou encore de la sociologie du droit (Commaille \& Jobert, 1999, Terré \& Frison-Roche, 1999). La seconde idée retient la tendance qu'ont les acteurs économiques eux-mêmes à préférer aux confrontations directes l'intervention d'instances de régulation, et notamment du législateur — qui n'est que l'une d'entre elles pour régler les modalités de la concurrence qu'ils se livrent. En atteste l'émergence, à la demande des opérateurs économiques eux-mêmes, d'un droit de la concurrence en réaction aux phénomènes de concentration (Boy, 2004), ou encore, l'histoire de la lutte contre les fraudes commerciales en France (Féral, 1995) et notamment l'épisode de la loi de 1905 sur la répression des fraudes (Canu \& Cochoy, 2004 ; Kessous, 2004 ; Stanziani, 2003b).

Pourtant, la publicité comparative vient bousculer quelque peu ces conceptions des rapports entre droit et marché, dans la mesure où la régulation instaurée par le droit autorise ici une concurrence des plus frontales entre deux acteurs marchands tout en définissant très précisément et de l'extérieur, les conditions d'exercice de cette confrontation. Rappelons que la publicité «en général» est considérée comme un outil indispensable au producteur pour se faire connaître en tant qu'acteur de l'offre, rendre visibles ses produits et se démarquer des

7. Pour une présentation approfondie du droit économique et de l'analyse économique du droit Cf. (respectivement) Farjat (1992) et Kirat (2000).

8. On peut sans doute considérer avec Richard Swedberg que la nouvelle sociologie économique a longtemps ignoré la loi et plus précisément les rapports que cette dernière entretient avec l'économie (Swedberg, 1997, p. 254). Pourtant, non seulement des travaux récents soulignent l'encastrement institutionnel et juridique du marché (Le Velly, 2002), mais ce courant soutient également depuis longtemps que «les marchés ne sont pas le résultat d'un ordre spontané d'agents économiques cherchant à optimiser les formes de leurs transactions marchandes ; ces derniers sont le résultat d'un ensemble non coordonné de décisions institutionnelles (politiques, juridiques, économiques), de relations personnelles et culturelles subissant les contingences de l'histoire [...] le contexte social dans lequel sont encastrées les relations marchandes est donc crucial » (Steiner, 1999, p. 73). 
entreprises concurrentes et de leur production ; pourtant la publicité comparative, loin de faire oublier les concurrents en se focalisant sur la seule offre de l'annonceur, place l'un d'entre eux au cœur du message. Un tel procédé constitue un parfait contre-pied à la doctrine publicitaire, dans la mesure où l'on accorde ici à un adversaire direct le rôle de co-vedette dans une annonce censée promouvoir le bien ou le service pour lequel la campagne est réalisée. En contrepartie, la publicité comparative sort les concurrents de l'anonymat supposé des relations marchandes et de la menace à la fois pesante et vague du marché, pour les engager dans un jeu plus « goffmanien» au cours duquel le vaincu court le risque de «perdre la face». Aussi, faire partager la vedette au produit concurrent est-il surtout un moyen employé dans l'espoir de sortir vainqueur et valorisé d'une confrontation valorisante (la comparaison est souvent organisée par un challenger qui veut se mesurer aux «favoris », aux leaders d'un marché, comme le montre bien l'exemple de la téléphonie avec l'affrontement entre Télé2 et France Telecom, $C f$. infra) en jouant sur les forces et les faiblesses de chacun. On conçoit ainsi qu'une comparaison publicitaire libre de toutes contraintes juridiques et déontologiques porterait probablement atteinte au bon fonctionnement du marché : cette rhétorique publicitaire comparative semble en effet se jouer de catégories, tels que le mensonge, le dénigrement, ou la loyauté, auxquelles les juristes se montrent pourtant particulièrement sensibles.

Aux paradoxes qui entourent la publicité comparative comme pratique marchande succède donc la double hésitation des législateurs et des juges quant à sa régulation et son application. Nous proposons, avant d'aborder les incertitudes rencontrées par les acteurs économiques et juridiques au moment de mobiliser la règle et de l'appliquer, de retracer l'histoire juridique de la publicité comparative en France ${ }^{9}$. L'anamnèse à opérer n'est pas très ardue : dans le droit français, le texte de loi fondateur en matière de publicité comparative ne date que de $1992^{10}$. Les tergiversations du législateur en la matière et l'élaboration chaotique de textes régulant cette pratique nous poussent néanmoins à remonter davantage le fil de l'histoire. Quatre périodes nous semblent pouvoir être distinguées.

Durant la première, qui s'étend jusqu'au début des années 1980, la jurisprudence, alors seule source de droit, considère comme strictement illicite toute publicité dans laquelle l'annonceur fonde son message sur une comparaison des caractéristiques des produits. Pour légitimer leur décision, les juges font appel à trois textes dont aucun ne concerne directement la publicité comparative : l'article 1382 du code civil (qui sanctionne la concurrence déloyale et le dénigrement), l'article 422 deuxième du code pénal (qui sanctionne l'utilisation et la citation de marque sans autorisation de son titulaire), l'article 44-I de la loi «Royer » du 27 décembre

9. La situation de la publicité comparative diffère largement selon les pays, notamment entre les Etats-Unis et l'Europe : non seulement la part des publicités comparatives est très largement supérieure aux États-unis (25 \%), mais la charge de la preuve y est aussi inversée : « in the European Union the starting point is prohibition, whereas in the United States a comparative advert is presumed acceptable until proven otherwise » (Spink et Petty, 1998, p. 867). Pour une présentation certes ancienne mais très fouillée du cas américain, $C f$. Sterk, 1976.

10. Ce qui ne signifie pas pour autant qu'il faille mésestimer l'ancienneté de l'usage de la rhétorique comparative pour présenter les offres : «[en 1229] une lettre "publicitaire" vantant les qualités de la nouvelle université de Toulouse créée autoritairement par le représentant du pape [...] informe sur les matières enseignées et n'hésite pas à se lancer dans une publicité comparative avec la plus grande université du royaume : celle de Paris. À Toulouse, les maîtres sont plus zélés et assidus qu'à Paris (où une grève à débuté en 1229) ; de plus les livres qui y sont interdits (certains livres d'Aristote) sont étudiés à Toulouse » (Polo de Beaulieu 2004, p. 15). 
1973 (qui sanctionne les atteintes aux principes de loyauté et de véracité de la publicité). Ce déficit de cadres juridiques formels est probablement la principale cause de l'inflexibilité dont font preuve les juges au moment de trancher les conflits, si l'on considère avec eux qu'un verdict autorisant la publicité comparative peut «faire jurisprudence » et «ouvrir une brèche » dans laquelle risquent de s'engouffrer par la suite tous ceux qui souhaitent user et abuser d'une forme de publicité perçue comme dangereuse.

Les modifications que la chambre de commerce internationale apporte au code des Pratiques Loyales en Publicité en 1966 et 1973 sont les premiers signes d'une acceptation partielle de la publicité comparative (Dianoux, 1999). Ces modifications, qui ouvrent une seconde période, interviennent au moment où le consumérisme s'institutionnalise, avec par exemple le lancement du journal Que choisir en 1961, la création de l'INC en 1968, etc. (Pinto, 1990 ; Cochoy, 2002b). L'acceptation progressive de la publicité comparative s'inscrit donc dans le prolongement des essais comparatifs de produits et autres guides d'achat (Karpik, 2000 ; Mallard, 2000a ; Cochoy, 2002a), dont cette pratique tente de capter la légitimité ${ }^{11}$. Il faut néanmoins attendre la fin des années 1970, pour que l'illicéité juridique de la publicité comparative fasse l'objet de controverses auxquelles vont prendre part non seulement les acteurs du marché, mais aussi les acteurs des sphères politique et juridique. Malgré les réticences affichées par les annonceurs et les publicitaires, inquiets des risques de dénigrement des marques et d'une indécision accrue chez les consommateurs, la cristallisation de l'attention autour de la publicité comparative se traduit par un courant favorable à un assouplissement de différentes régulations qui l'encadrent: les pouvoirs publics (le rapport «Scrivener» de 1978) et les grandes associations de consommateurs (Lascombes \& Legeret, 1999) encouragent, sous réserve, l'acceptation juridique de cette forme de publicité. Sans doute, aucun des deux projets de loi proposés durant cette période ne sera adopté — le projet de loi Scrivener en 1978 d'abord, puis celui de Catherine Lalumière en 1984 (Dianoux, 1999). En revanche, cette dynamique partisane se traduit au niveau des tribunaux par une jurisprudence de juillet 1986 qui représente une date clé dans cette histoire. À propos d'une affaire opposant deux distributeurs (Carrefour versus SRGM Groupe Arlaud) sur des questions de comparaison de prix, la chambre commerciale de la cour de cassation autorise pour la première fois la publicité comparative sous quatre conditions : a) la comparaison doit porter sur des produits identiques ; b) les produits doivent être vendus «dans les mêmes conditions, par des concurrents différents »; c) le message publicitaire doit se limiter à une publication de prix ; d) l'exactitude des indications données doit être incontestable (Cass. com., 22 juill. 1986). Un tel verdict est décisif en ce qu'il ose ce qu'aucun autre n'avait osé avant lui : « entrouvrir la brèche » dans les digues très hermétiques qui empêchaient jusqu'alors tout déferlement de la publicité comparative. Il apparait à la fois comme l'aboutissement du courant favorable que nous venons d'évoquer, mais aussi comme une préfiguration des transformations juridiques à venir.

11. Notons que la publicité propose une «extension-restriction» des essais comparatifs, d'où peut-être l'attitude ambiguë des milieux consuméristes à son égard (Douriez, 2003). La publicité comparative étend la logique des essais comparatifs, dans la mesure où les campagnes associées se font à une échelle beaucoup plus vaste que l'espace des guides et des journaux consuméristes, mais elle en restreint la portée, dans la mesure où la comparaison opérée est le plus souvent bilatérale et non multilatérale, faiblement outillée, et où le choix des critères et des méthodes de comparaison est entièrement à la discrétion du «compareur » (et du droit qui encadre sa pratique). 
La troisième période s'ouvre avec la promulgation de la loi du 18 janvier 1992 — qui vise à renforcer la protection du consommateur —, dont l'article 10 donne lieu à l'introduction des articles L121-8 à L121-14 dans le code de la consommation. Ces articles, suivant en cela le sens de la jurisprudence qui les précède, autorisent pour la première fois au niveau légal le maniement et l'utilisation de la publicité comparative, pourvu qu'elle s'insère dans les cadres très stricts prévus à son effet. Elle est définie comme "la mise en comparaison des biens ou services en utilisant soit la citation ou la représentation de la marque de fabrique, de commerce ou de service, de la raison sociale ou de la dénomination sociale du nom commercial ou de l'enseigne d'autrui. L'identification du comparé devait donc être explicite » (Wilhelm \& Dubarry ; 2002). Cette définition exclut de la catégorie toutes les comparaisons entre le produit et l'ensemble du marché ou une forme générique de produit concurrent; la comparaison ne peut en effet porter que sur deux produits à l'identité clairement identifiée par l'annonceur et identifiable pour le consommateur, ce qui ajoute encore à l'impression de violence symbolique dégagée par cette pratique. Les annonceurs sont également conviés à entrer avec la plus grande précision et «objectivité » possibles dans le détail, dans la composition du produit ou du service. La comparaison doit en effet être «objective et ne peut porter que sur des caractéristiques essentielles, significatives, pertinentes et vérifiables de biens ou services de même nature disponible sur le marché ». En d'autres termes la nouvelle définition juridique de la publicité comparative pousse désormais, en quelque sorte, les professionnels du marché à dépasser la qualité au sens d'Akerlof (une qualité globale et/ou moyenne qui qualifie le produit dans son ensemble) pour pénétrer dans le produit et la combinaison de ses caractéristiques telles que les conçoit Lancaster (une qualité plurielle qui résulte de la combinaison d'un ensemble de caractéristiques). De ce point de vue, l'annonceur doit aussi accepter de changer de registre, de faire de la publicité autrement, en renversant les proportions habituelles de ses composants : alors que dans une publicité classique il adjoint le plus souvent au symbolique et au rêve un soupçon de réalité, il ne peut dans une publicité comparative, à l'inverse, ajouter à la réalité qui constitue l'essentiel du message qu'un zeste de rêve, d'imaginaire, pour autant que ce rajout soit juridiquement acceptable. Parce qu'elle emprunte le ton de l'objectivité et de la neutralité pour apporter aux consommateurs des preuves irréfutables de la supériorité de l'offre qu'elle met en scène, la publicité comparative se rapproche d'un dispositif de jugement (Karpik, 1989) tel que le guide d'achat, à l'autorité supérieure, capable de gagner la confiance des consommateurs en prenant précautionneusement appui sur certains attributs du produit pour établir une comparaison considérée comme objectivement valable. Or la forme « désintéressée » de la comparaison, déjà discutable dans le cas des guides d'achat (Cochoy, 2002a), s'efface bien sûr ici au profit de l'intérêt bien compris de l'annonceur. Au final, il convient de retenir que le droit va accompagner cette première définition d'un tel nombre de conditions, que cette autorisation partielle ne tranche pas avec l'interdiction qui prévalait jusque-là. En d'autres termes, « Les publicités ne semblent guère plus nombreuses depuis 1992 qu'avant l'entrée en vigueur du texte »(Cas \& Lappare, 2001 ; p. 2902) ${ }^{12}$.

12. Sans adhérer à la thèse qui voudrait que cette loi, par le nombre de restrictions imposées, soit avant tout destinée à empêcher la publicité comparative, il est facile de constater que la dizaine de conditions associées à son maniement viennent assez largement en réduire l'usage ; $C f$. notamment l'obligation de «[communiquer] l'annonce comparative aux professionnels visés, dans un délai au moins égal à celui exigé, selon le type de support retenu, pour l'annulation d'un ordre de publicité » (Code de la consommation, article L 121-12) ou encore le fait que la publicité «doit être limitée à une comparaison objective qui ne peut porter que sur des 
L'adaptation de notre législation à la directive européenne 97/55/CE du 6 octobre 1997 est le point de départ de la quatrième et dernière étape ; il faut attendre août 2001 pour que cette transposition ait lieu et modifie les articles L121-8 à L121-12 du code de la consommation, en encadrant désormais la publicité comparative de la façon suivante :

«Toute publicité qui met en comparaison des biens ou services en identifiant, implicitement ou explicitement, un concurrent ou des biens ou services offerts par un concurrent n'est licite que si :

$1^{\circ}$ Elle n'est pas trompeuse ou de nature à induire en erreur ;

$2^{\circ}$ Elle porte sur des biens ou services répondant aux mêmes besoins ou ayant le même objectif ;

$3^{\circ}$ Elle compare objectivement une ou plusieurs caractéristiques essentielles, pertinentes, vérifiables et représentatives de ces biens ou services, dont le prix peut faire partie.

Toute publicité comparative faisant référence à une offre spéciale doit mentionner clairement les dates de disponibilité des biens ou services offerts, le cas échéant la limitation de l'offre à concurrence des stocks disponibles et les conditions spécifiques applicables.» (Code de la consommation, article L121-8)

«La publicité comparative ne peut :

$1^{\circ}$ Tirer indûment profit de la notoriété attachée à une marque de fabrique, de commerce ou de service, à un nom commercial, à d'autres signes distinctifs d'un concurrent ou à l'appellation d'origine ainsi qu'à l'indication géographique protégée d'un produit concurrent ;

$2^{\circ}$ Entraîner le discrédit ou le dénigrement des marques, noms commerciaux, autres signes distinctifs, biens, services, activité ou situation d'un concurrent ;

$3^{\circ}$ Engendrer de confusion entre l'annonceur et un concurrent ou entre les marques, noms commerciaux, autres signes distinctifs, biens ou services de l'annonceur et ceux d'un concurrent ; $4^{\circ}$ Présenter des biens ou des services comme une imitation ou une reproduction d'un bien ou d'un service bénéficiant d'une marque ou d'un nom commercial protégé.» (Code de la consommation, article L121-9)

«[...] l'annonceur pour le compte duquel la publicité comparative est diffusée doit être en mesure de prouver dans un bref délai l'exactitude matérielle des énonciations, indications et présentations contenues dans la publicité. » (Code de la consommation, article L121-12)

De l'aveu même de professionnels du droit, si les modifications du texte ont élargi le champ d'application de la publicité comparative, elles n'ont pas entraîné de grands bouleversements dans les tribunaux dans la mesure où les juges se montraient déjà sensibles, depuis 1997, au nouveau positionnement du droit européen vis-à-vis de cette pratique et faisaient une interprétation plus large des textes français (Wilhelm \& Dubarry, 2002). Assurément, les différences entre les deux textes sont loin d'être frappantes ; l'hésitation est toujours de mise : le nouveau texte continue à restreindre très fortement ce qu'il prétend autoriser par ailleurs. Mais certains indices témoignent néanmoins d'un assouplissement de la règle de droit: premièrement, l'identification implicite du concurrent est désormais autorisée là où le texte de 1992 ne permettait qu'une identification explicite ${ }^{13}$. Seconde modification : les biens visés ne doivent plus être de «même nature » mais répondre «aux mêmes besoins ou [aux] mêmes objectifs »; cette nouvelle rédaction, selon les auteurs du texte beaucoup moins restrictive que la précédente, rendrait par exemple possible la comparaison des prix d'un trajet Paris/Toulouse

caractéristiques essentielles, significatives, pertinentes et vérifiables de biens ou services de même nature et disponibles sur le marché » (Code de la consommation, article L 128-8).

13. Notons immédiatement que la jurisprudence semble appelée à conserver un rôle majeur autour de l'interprétation d'une notion aussi floue, qui vient encore accroître l'incertitude autour du jugement, et ajouter à la difficulté de la tâche du juge. En attestent les propos tenus par Pascal Wilhelm (Avocat à la Cour) et Marie Dubarry (juriste) : «Une incertitude demeure quant à l'appréciation de cette identification implicite : faut-il faire appel à la notion de "consommateur moyen" pour apprécier la réalité de l'identification de la marque d'un concurrent ? La jurisprudence à venir nous le dira » (Wilhelm \& Dubarry, 2002). 
en train avec le même trajet en avion (ibid.). Troisièmement, la comparaison peut désormais être légalement valable alors qu'elle ne porte que sur une seule des caractéristiques du produit ou du service, pourvu néanmoins que cette caractéristique soit pertinente, vérifiable et représentative. La quatrième modification est de taille: il s'agit de la suppression des dispositions particulièrement contraignantes qui exigeaient la communication préalable de la publicité comparative aux professionnels visés. Ces deux dernières modifications figurent parmi celles qui avaient déjà été anticipées par certains tribunaux (ibid.). Le reste des dispositions ne trahit en revanche en rien la version d'origine - le parasitisme et le dénigrement sont, bien sûr, toujours condamnés avec la même vigueur — et si d'autres modifications terminologiques mériteraient d'être relevées ${ }^{14}$, nos remarques précédentes nous amènent à insister sur le faible écart, sur le papier, entre la portée du texte de 2001 et celle du texte de 1992 - qui lui-même n'avait, en son temps, pas révolutionné les pratiques telles que les acceptait déjà la jurisprudence.

Que pouvons-nous finalement retirer de cette histoire juridique de la publicité comparative et de notre découpage en quatre temps ? Tout au plus avons-nous relevé de légères retouches successives qui, pour les non-spécialistes du droit, s'avèrent plutôt décevantes. Même les professionnels du droit, dans leurs analyses ou leurs propos, donnent le sentiment de voir défiler une histoire où, malgré une tendance confirmée vers un assouplissement des règles et des pratiques juridiques ${ }^{15}$, la publicité comparative apparaît quels que soient les cadres en vigueur comme dangereuse pour les acteurs du marché, particulièrement limitée par le droit, et au final bien peu utilisée par les annonceurs. Néanmoins, dans le détail, l'histoire de la publicité comparative est surtout celle d'une hésitation constante et d'une invention continue de la jurisprudence la concernant. Cette hésitation nous semble être le symptôme d'un problème et d'un usage. C'est le symptôme d'un usage, puisque la jurisprudence renvoie à des pratiques et à des litiges ; c'est aussi le symptôme d'un problème, puisque les atermoiements de la jurisprudence témoignent des difficultés qu'éprouvent les juges, dans le cas précis, à établir la correspondance entre les règles de droit et les cas qui leur sont soumis. C'est donc autour de ces deux points que nous proposons d'éclaircir les enjeux de la comparaison publicitaire : dans la deuxième section de ce texte, nous examinerons la publicité comparative non plus du côté des textes formels et de leur révision, mais du côté des pratiques réelles et de leur examen. Cet examen met en lumière l'espace d'une double hésitation/expérimentation juridique et marchande quant à l'opportunité de mobiliser la règle et/ou de jouer avec le droit.

\section{Des hésitations à mobiliser la règle : quand les acteurs jouent avec le droit}

Si le droit hésite vis-à-vis de la publicité comparative, c'est peut-être parce que cette pratique, même cadrée juridiquement, semble contrevenir à l'adage fondateur de l'autorité du droit d'après lequel «nul ne peut se faire justice à soi-même». Il est en effet possible de

14. Par exemple ces quelques changements de termes dont les futures jurisprudences diront s'ils sont ou non anodins : caractéristiques significatives en 1997 devient caractéristiques représentatives en 2001 ; nécessité pour l'annonceur de pouvoir prouver «l'exactitude matérielle des données de fait» et non plus «l'exactitude de ses allégations ».

15. Depuis les assouplissements juridiques de 2001, la publicité comparative semble gagner du terrain chez les annonceurs et les publicitaires (Girard, 2003). 
considérer que la publicité comparative amène ceux qui s'y livrent à «se faire justice à euxmêmes », si l'on veut bien prendre ce terme de «justice » au sens du modèle de la justification (Boltanski \& Thévenot, 1991) : les concurrents qui recourent à ce type de publicité s'affrontent pour faire valoir leur «valeurs» (ou grandeurs), en référence aux ordres de justification industriel et/ou marchand (qualité, prix, service...). Mais ici, l'appel à la «justice » s'exerce (au moins dans un premier temps) d'une façon très particulière, qui consiste à réactiver, sous une forme distante et technicisée, un rapport social que l'on aurait pu croire à jamais disparu et proscrit par le droit: le duel. Tel un duelliste, l'entreprise qui convoque son ou ses concurrent(s) dans une publicité comparative n'attend pas de s'en remettre à la décision d'un juge pour faire valoir son argument, mais affronte directement son concurrent sur le « champ d'honneur » du marché, en prenant l'ensemble du public à témoin ${ }^{16}$. D'un côté, cette façon de faire est encore plus agressive qu'un duel classique, puisque la publicité comparative permet à l'un des protagonistes de forcer la participation du second au duel, et de décider seul du choix des armes (ici les critères d'après lesquels s'opère la comparaison). Mais d'un autre côté, la publicité comparative est plus civile, puisqu'elle consiste à régler ce duel en passant par le droit : cette fois la pratique s'oriente d'emblée (ou peut s'orienter) d'après sa codification par l'ordre juridique disponible. Toutefois, face à un droit hésitant, les acteurs hésitent eux-mêmes à s'engager dans une voie truffée de pièges et d'embûches. Pour avancer dans cet ordre juridique incertain, deux chemins s'offrent à eux — deux chemins que nous voudrions maintenant explorer.

La première façon de se confronter directement à son (ou ses) concurrent(s) en référence au droit se manifeste paradoxalement lorsque cette confrontation directe est perçue ou posée comme juridiquement impossible. Pour illustrer ce cas de figure, on peut citer l'exemple d'une publicité de Peugeot réalisée en 2004. L'affiche met en scène deux véhicules sur une même route, dans un même virage : le premier, sortant fièrement de la courbe face à nous, bien à sa place, est une Peugeot 407 ; le second, légèrement en retrait, gît en revanche à l'extérieur du tournant, les quatre pneus en l'air, non loin d'une dépanneuse située à l'arrière plan. Sur le châssis de l'épave on peut lire un bossage en gros caractères : "Basic toy car ». Ce visuel présente toutes les apparences d'une publicité comparative entre deux produits : Peugeot 407 vs. Basic toy car. Sans doute, la comparaison établie vise un produit fictif - une «voiturejouet de base » introuvable sur le marché, sauf peut-être en modèle réduit. Mais dans le visuel ce produit fictif, loin de se présenter comme une catégorie abstraite, se donne plutôt à voir sous la forme d'un objet incarné, matériel, tangible : même en l'absence de comparant réellement identifiable on est bien en présence de l'imagerie classique de la publicité comparative, c'est-àdire de la mise en équivalence de deux produits singuliers présentés comme «palpables », grâce au truchement d'une infographie représentant deux voitures d'apparence «réelle », de taille comparable, «photographiées » en trois dimensions.

16. Notons que lorsque Max Weber a abordé la question du possible conflit entre différents ordres de normes sociales, c'est précisément le duel qu'il a pris comme exemple (le recours au duel manifeste l'opposition entre l'honneur et le droit). Mais, à la différence de Weber pour qui la résolution d'un tel conflit pouvait être trouvée en procédant de façon séquentielle — se battre d'abord en duel pour sauver son honneur puis se rendre aux autorités pour manifester son respect du droit (Weber, 1995, p.66) - la publicité comparative invente une mise en compatibilité certes délicate mais simultanée des principes qui fondent l'opposition entre la raison marchande (qui remplace ici la défense de l'honneur) et le respect du droit. 
Ici, tout se passe comme si l'existence des restrictions juridiques qui pèsent sur la publicité comparative était à la fois connue, admise, respectée, mais aussi tournée ${ }^{17}$. D'un côté, cette publicité mobilise une hésitation possible entre le même et le même — deux voitures comparables - pour mieux souligner le caractère illusoire d'une telle hésitation: une Peugeot 407 n'est pas une voiture ordinaire. Cette publicité reprend la figure de l'âne de Buridan chère aux acteurs du marché : capter une clientèle, c'est d'une part suggérer une hésitation possible entre le même et le même, grâce à la mise en parallèle visuelle de deux produits tangibles, et d'autre part trouver le moyen de lever aussitôt cette indécision, en soulignant « la différence qui convient» (Cochoy, 2002a). Or n'est-ce pas parce que la loi rend très difficile, voire périlleux, d'orienter la comparaison entre deux produits réels sans l'enfreindre que les acteurs cherchent ici le moyen de comparer sans comparer, via l'introduction d'un comparant d'apparence réelle mais néanmoins fictif? En ne visant ni explicitement ni même implicitement «un» concurrent particulier, un tel procédé tente de s'abstraire des situations de comparaison strictement définies par le droit ( $C f$. supra) ; tout se passe comme si Peugeot, avec son infographie, cherchait le moyen de surmonter l'hésitation entre la publicité comparative au risque de la sanction juridique et l'absence de publicité comparative au risque de se priver de son rendement économique supposé.

Cette astuce a en effet pour résultat ironique de multiplier à l'infini ces duels que la loi tente de canaliser. En figurant la comparaison entre un «produit générique objectivé » et le produit vanté par l'annonceur, le dispositif publicitaire invite le chaland à faire ce qu'il ne fait pas luimême : il porte le public à compléter l'affiche en imaginant l'identité du ou des concurrents possibles ${ }^{18}$. Au bout du compte, la répétition des expectations diverses face au spectacle de l'affiche finit par suggérer la supériorité du produit comparé face à l'ensemble des comparants possibles, et par laisser à l'annonceur une entière liberté dans le choix des critères susceptibles de fonder cette supériorité (fiabilité et solidité pour Peugeot par exemple), puisque rien n'est «mesurable» en matière de fiction. Mais il y a plus: le jeu qu'engagent les acteurs économiques autour de la règle de droit en manifeste paradoxalement l'existence et la prégnance. Ou bien la loi est mise à distance, tournée ou contournée, et dans ce cas le geste publicitaire souligne en creux la force de la règle, un peu comme le duelliste chez Weber manifeste, lorsqu'il choisit de dissimuler son geste, sa pleine reconnaissance de la norme (Weber, 1995, p. 66). Ou bien les acteurs violent la règle, passent outre, engagent des comparaisons à leurs risques et périls, et dans ce cas leur action crée les conditions nécessaires au rappel de la règle de droit, un peu comme le crime chez Durkheim autorise la réaffirmation de la conscience collective (Durkheim, 1986). L'exercice quotidien du marché est donc bien au fondement même de l'institution juridique, sans laquelle il ne peut exister.

La deuxième façon de se comparer via la médiation du droit repose au contraire sur une action a priori étroitement conforme à ses prescriptions, comme semble s'y être appliquée (par exemple) une publicité comparative récente de Télé2 à l'encontre de France Telecom parue dans la presse ${ }^{19}$. Cette publicité part d'une proposition en lettres capitales — « TÉLÉPHONE

17. Pour une présentation juridique de cette stratégie dans le contexte américain, $C f$. Sterk, 1976.

18. Pour une sociologie économique stimulante du renouveau de la notion de produit générique dans le marché contemporain, $C f$. Nouguez, 2004.

19. Metro Toulouse, vendredi 4 mars 2005, n²55, p. 16. 
ILLIMITÉ : VOUS N'AUREZ BIENTÔT PLUS QU'UNE OREILLE », et joint aussitôt l'image au slogan: le haut de la page est occupé par la photo d'un écrin à bijoux ouvert, mais dans lequel on ne voit qu'une seule boucle d'oreille, le deuxième emplacement restant vide. Suit le tableau comparatif suivant ${ }^{20}$ :

\begin{tabular}{|c|c|c|}
\hline $\begin{array}{c}\text { TOUS VOS APPELS } \\
\text { ILLIMITÉS }^{(1)}\end{array}$ & $\begin{array}{c}\text { TELE2 } \\
\text { 24H] } 24 \text { et } 7 \mathrm{~J} / 7\end{array}$ & $\begin{array}{c}\text { FRANCE TELECOM } \\
{\left[\mathrm{X}^{\prime}\right] €}\end{array}$ \\
\hline $\begin{array}{c}\text { VOS 3 NUMÉROS } \\
\text { PRÉFÉRÉS ILLIMITÉS }\end{array}$ & {$[\mathrm{y}] €$} & {$\left[\mathrm{y}^{\prime}\right] €$} \\
\hline
\end{tabular}

L'affiche se poursuit en introduisant deux précisions : l'une signale l'existence d'autres produits indépendants de la comparaison — «Découvrez nos autres forfaits illimités sur www.tele2.fr et profitez également de nos tarifs vers les mobiles et l'international » — l'autre complète les conditions de l'offre : «En plus vous restez libre : il n'y a aucun engagement de durée et vous conservez le même numéro de téléphone ». Enfin, l'affiche mentionne un numéro d'appel gratuit (en gros caractères) que l'on peut composer pour s'inscrire, puis reproduit le logo de l'entreprise et sa devise : «pourquoi continuer à téléphoner trop cher ?» Tout en bas suivent les petits caractères explicitant les deux notes du tableau ( $C f$. infra) et, en haut à droite — pour ne rien oublier ! — on trouve verticalement (en caractères minuscules) le nom de l'agence de publicité : J. Walter Thompson ${ }^{21}$.

Cette fois-ci, la publicité comparative n'est manifestement pas perçue ou présentée comme impossible et la loi ne fait a priori l'objet d'aucun contournement - au contraire, elle paraît strictement appliquée. À première vue, il semble bien que l'annonceur, conformément aux termes de la loi, se réfère à des «biens ou services répondant aux mêmes besoins ou ayant le même objectif » (la comparaison porte sur les prix de deux services téléphoniques identiques : des forfaits «illimités», sept jours sur sept et vingt-quatre heures sur vingt-quatre) et « compare objectivement une ou plusieurs caractéristiques essentielles, pertinentes, vérifiables et représentatives de ces biens ou services, dont le prix peut faire partie » (ici la comparaison est restreinte au prix). Tout se passe comme s'il n'y avait ni parasitisme ni confusion possibles (les attributs de France Telecom — logo et marque — n'apparaissent pas, et le comparant et le comparé sont clairement distingués) ; on ne note pas non plus de dénigrement apparent (aucune appréciation n'est portée sur France Telecom), etc.

Sans doute, la vigilance chère au sociologue demande de ne pas aller trop vite en besogne, de scruter l'affiche dans ses moindres recoins, de prendre une loupe pour lire tous les petits

20. Nous avons masqué les prix dans la mesure où notre propos porte sur la forme de la comparaison et non sur son contenu, et ne consiste évidemment pas à faire de la publicité pour quelque opérateur que ce soit.

21. Les spécialistes de l'histoire des marchés (Strasser, 1989 ; Chessel, 1998) savent à quel point cette agence a joué un rôle à la fois pionnier, majeur et moteur dans l'histoire de la publicité contemporaine. Le fait que ce soit une agence aussi importante pour l'identité et l'orientation de la profession publicitaire qui vienne aujourd'hui soutenir le développement de la publicité comparative en France ne saurait donc être considéré comme tout à fait anodin. 
caractères afin d'y traquer l' « arnaque » ou l' «illusion» que l'anthropologie des marchés aime à débusquer derrière les séductions des surfaces marchandes (Duval, 1981; de la Pradelle, 1996). Mais dans le cas précis, l'ironie veut que la mobilisation de cette sage suspicion débouche plutôt sur un résultat contraire à ses attentes de prédilection : il semble qu'ici les «notes de bas d'affiche » visent davantage à prémunir l'opérateur téléphonique contre les ruses des consommateurs (de Certeau, 1980) qu'à tromper ces derniers ; Télé2 chercherait peut-être moins à abuser ses clients qu'à se protéger de clients susceptibles d' «abuser » de la téléphonie illimitée, par exemple en tentant de détourner cette offre de communication vers un usage professionnel, vers le partage du forfait entre plusieurs foyers, et/ou vers un accès gratuit à l'Internet bas débit offert par d'autres opérateurs sur le réseau téléphonique : n'est-ce pas pour prévenir de tels détournements d'usage que les mentions portées en bas d'affiche précisent d'une part que l'offre est «réservée aux particuliers, exclusivement pour un usage personnel et familial », et d'autres part que ces appels s'entendent «hors numéros spéciaux, hors numéros d'accès à Internet et hors télécopies » ? Pour le reste ces derniers services «non compris dans le forfait » sont signalés comme étant «facturés selon le tarif de base de TELE2 en vigueur », c'est-à-dire, en termes économiques, toutes choses égales par ailleurs, de sorte qu'il semble bien difficile de trouver ici l'arnaque censée se dissimuler habituellement du côté des petits caractères.

Nous nous demanderons plus loin, avec les acteurs, si une publicité comparative «publiée » est toujours conforme aux règles de droit et/ou exempte de tromperie (mais aussi, et symétriquement, si ces règles sont aussi lisibles qu'on pourrait le présumer). Mais à supposer que ce soit le cas, est-ce à dire que la loi reste extérieure au jeu économique et se contente de border sagement le jeu des acteurs ? Que nenni. D’abord, la rareté même du procédé en radicalise l'audace et l'importance. La comparaison des forfaits téléphoniques engage non seulement deux acteurs du marché - Télé2 et France Telecom - mais aussi le droit luimême, qui fait implicitement partie de la rhétorique publicitaire. Si la publicité comparative est si restrictive, si certains acteurs comme Peugeot préfèrent la contourner, et si Télé2 y recourt néanmoins, c'est bien que l'opérateur privé cherche à mettre en scène son argument comme inattaquable: ses tarifs seraient plus avantageux que ceux de France Telecom. Ainsi la réticence politique de l'État à fournir certaines armes au jeu concurrentiel se retourne une fois de plus contre lui : l'acteur qui recourt à la publicité comparative en appelle non seulement au calcul économique du consommateur, mais aussi à la caution implicite du législateur, et donc au soutien indirect et involontaire de l'autorité juridique : le droit fonctionne ici comme un « label malgré lui ».

Bien entendu, dans le détail les choses sont plus complexes ; pour y voir tout à fait clair il conviendrait d'examiner l'autre hypothèse — instruire l'examen juridique de l'affiche, établir si la publicité est licite ou non. Pourtant, pas plus que nous n'avons voulu conclure en faveur de la première hypothèse, nous nous garderons d'engager l'instruction de la seconde. À quel titre pourrions-nous prétendre mieux juger de la légalité et de la loyauté des comportements que les plaignants et surtout que le juge professionnel censé les départager? Si un adage rappelle que « nul n'est censé ignorer la loi », un autre précise que c'est toutefois au juge et à nul autre que revient de dire le droit : «jurisdictio ». Sommés à la fois de ne pas se soustraire au droit et de s'en remettre aux décisions du juge, les acteurs n'ont donc d'autre choix que de faire l'hypothèse d'une «présomption » de conformité de leurs actes ou de leurs analyses à la 
lettre du droit; ainsi, une affiche sera «présumée » conforme au droit tant qu'elle n'aura pas fait l'objet de contestation. Or cette présomption de conformité est précisément, comme mus venons de le voir, la figure qui soutient toute la rhétorique de la publicité comparative : certes, c'est bien le juge qui juge, mais avant que la justice ne passe, la publicité convoque chacun comme juge, sur la légalité de la publicité comparative et sur la véracité de ce qu'elle dit.

Pourtant, cette présomption de conformité de la publicité au droit est fragile. Si le contrôle « en dernier ressort» de l'application du droit relève de la compétence du juge et non des acteurs, il suffit qu'un seul d'entre eux mobilise le juge pour que la présomption vacille, et que l'objet en cause soit soumis à l'examen de l'institution judiciaire. Or, dans le cas de l'affrontement entre Télé 2 et France Telecom, le juge n'a pas manqué d'être convoqué, et plutôt dix fois qu'une. La pratique de Télé 2 en matière de publicité comparative possède un intérêt et une particularité tout à fait remarquables. Alors que la quasi totalité des cas recensés dans la presse ces vingt dernières années sont des tentatives d'un jour (qui plus est rarement couronnées de succès) ${ }^{22}$, Télé 2 a ceci de particulier de recourir à la publicité comparative de façon récurrente... une récurrence d'autant plus étonnante qu'elle s'exprime en dépit d'attaques en justice tout aussi systématiques — et le plus souvent fructueuses ! — de la part du principal acteur mis en cause. France Telecom a assigné Télé 2 en justice en 2000, à propos d'une publicité comparative portant sur les communications hors département à plus de $52 \mathrm{~km}$ (cette campagne a fini par être partiellement ${ }^{23}$ condamnée au terme d'une longue procédure ${ }^{24}$ ), en 2001 à propos d'une publicité comparative télévisée sur le thème des vampires (le recours en référé a été rejeté mais Télé2 a ensuite été partiellement condamné sur le fond ${ }^{25}$ ), en 2002 à propos d'une campagne de presse et de télévision comparant la durée des communications obtenues pour un euro sur la base du forfait 3 heures de France Telecom (à nouveau Télé 2 a fait l'objet d'une condamnation partielle ${ }^{26}$ ), en 2003 à propos d'une campagne de presse massive comparant les tarifs des deux opérateurs (Télé2 a encore été partiellement condamné) ${ }^{27}$. Enfin, en 2004, au sujet d'un slogan jugé dégradant (d'où une nouvelle condamnation partielle) $)^{28}$.

22. À l'exception peut-être des Centres Leclerc qui ont en leur temps bravé le droit à plusieurs reprises (Doyère, 1991 ; Peyrot, 1992). Mais à cette époque le recours systématique à cette pratique visait davantage à provoquer le juge (dans l'espoir bien sûr d'obtenir par ce geste une image de « distributeur cons umériste ») qu'à jouer le jeu de la concurrence ordinaire sur un autre terrain et avec d'autres armes.

23. Contrairement au préjugé commun qui veut qu'on «gagne » ou «perde » un procès, la réalité semble plus nuancée : une plainte se découpe généralement en plusieurs griefs dont certains sont suivis par le juge et d'autres non, de sorte qu'il est souvent difficile d'assigner un « vainqueur » clair aux duels judiciaires.

24. Jugement du tribunal de Commerce de Versailles du 23 avril 2003.

25. Jugement du Tribunal de Commerce de Paris du 19 décembre 2001.

26. Jugement du Tribunal de Commerce de Paris du 17 septembre 2002, confirmé par la cour d'appel de Paris le 4 décembre 2002.

27. Jugement du Tribunal de Commerce de Paris du 7 mai 2003.

28 Jugement du Tribunal de Commerce de Paris du 16 juillet 2004. Au-dessus de l'habituel comparatif des tarifs entre les deux opérateurs, la publicité annonçait: «Encore client de France Telecom? Comme c'est dommage... ». Cette rédaction fut jugée « disproportionnée et dégradante » par le tribunal. 


\section{Des hésitations à appliquer la règle : quand vient le tour du juge}

Que nous révèle l'examen de ces affaires ? Avant de répondre à cette question, il nous faut préciser dans quelles conditions et comment nous comptons l'aborder. Nous nous interdirons de juger de l'opportunité et de la légalité des décisions de justice, au nom du respect du droit, qui interdit de les commenter, mais aussi des principes de méthode en vigueur en matière de sociologie des controverses, qui commandent de ne pas présumer de la vérité ou de la fausseté des positions en présence (Bloor, 1982). Mais cette posture de principe et ce choix de méthode ne nous laissent pas démunis, bien au contraire. Plutôt que discuter du bien fondé sur la forme ou sur le fond de la décision de justice, nous proposons de nous intéresser aux processus d'ajustements dont elle est le produit, en soulignant à partir de l'étude de la jurisprudence à quel point l'exercice du jugement repose non seulement sur l'examen de la conformité au droit du cas examiné, mais aussi, de façon plus inattendue, sur la mobilisation de conceptions particulières du jeu économique et des rationalités engagées. Notre attention prioritaire se portera sur le «tour du juge », au double sens de «moment » et de «savoir-faire » judiciaire : de même que la clôture des controverses scientifiques ne dépend pas uniquement des ressources mobilisées dans l'administration de la preuve et des rapports de force entre chercheurs, mais aussi du contrôle institutionnel exercé par les comités de lecture, le règlement des différends commerciaux ne repose pas seulement sur le rapport marchand, mais sur l'intervention experte des officiers de justice.

L'attention portée au travail du juge peut surprendre. A priori, cette activité correspond exactement à ce que l'on attend du juge et occupe bien évidemment l'essentiel des décisions rendues. Notons toutefois que l'application du droit, même pour le juge, n'a rien de mécanique, de prévisible et d'irréversible; l'hésitation qui prévalait du côté de l'histoire puis de l'engagement des acteurs se retrouve une fois encore du côté de la justice. Comme nous allons le voir, l'exercice de la justice est en effet une démarche très précautionneuse qui — à l'instar des politiques fondées sur le principe de précaution - consiste à avancer à tâtons pour sonder les états du monde, de façon à trouver un chemin sûr et viable parmi différentes options certes possibles, mais incertaines et risquées (Latour, 2000). Cette façon d'avancer se donne à lire à la fois longitudinalement, dans les mouvements de balancier qui s'observent du référé à l'instance suivante, et latéralement, dans les ajustements que le juge opère entre les textes disponibles et les principes généraux du droit, ses propres conceptions et celles de ses collègues ${ }^{29}$, les éléments du dossier, les plaidoiries des avocats et les diverses représentations disponibles de l'économie. Si le juge dit le droit, il ne le dit pas tout seul : le juge s'appuie sur les aspérités du cas soumis, sur les prises que lui tendent ses collègues (de couloir en couloir - officieusement - ou d'instance en instance - officiellement), sur les productions du législateur mais aussi sur celles des acteurs de terrain (notamment les argumentaires des avocats, qui sont résumés dans chacun des jugements et parfois repris, nuancés ou discutés dans les attendus, explicitement ou implicitement). La prononciation d'un jugement est donc bien une diction

29. Emmanuel Lazega et Lise Mounier (2003) ont bien montré que la collégialité est l'un des modes privilégiés de gestion de l'incertitude pour les juges consulaires : «En situation d'incertitude, [le juge du Tribunal de commerce] est confronté à des flots d'information dont il ne contrôle pas toujours seul la pertinence. Ces incertitudes peuvent être partiellement contrôlées par des échanges d'idées et de conseils. » 
collective : dans les tribunaux, le droit s'écrit « ensemble », au carrefour des règles, des acteurs et des argumentaires.

Certes, illustrer chacun des points que cette dernière remarque soulève excèderait à la fois l'espace disponible ici et notre souci d'interroger la publicité comparative du point de vue de la sociologie économique. Aussi, parmi l'ensemble des instances qui participent à la polyphonie juridique, nous contenterons-nous d'étudier l'intervention des conceptions économiques en vigueur dans le travail des avocats d'une part et du juge d'autre part. Pour ce faire, nous examinerons à titre principal ${ }^{30}$ une affaire portant sur un spot publicitaire de Télé 2 mettant en scène un vampire mourant au téléphone, pour avoir téléphoné « trop cher » en plein jour (sousentendu: au tarif «heures pleines » de France Telecom). Paradoxalement, cette affaire est d'autant plus exemplaire qu'elle est atypique. L'affaire est atypique du point de vue qui nous préoccupe, à savoir souligner et étudier les liens que la publicité comparative entretient avec les catégories de l'économie. C'est même le pire exemple que l'on puisse retenir pour éclairer de tels liens, puisque le spot en question met en scène un vampire, auquel l'opérateur historique serait implicitement assimilé ${ }^{31}$. Au lieu de se limiter au type de correspondance précise et rationnelle entre biens ou services comparables que nous avons jusqu'ici présenté comme principal attribut de la publicité comparative, voilà que le spot de Télé2 mobilise tout le pouvoir d'évocation symbolique de la publicité traditionnelle, pour le retourner ici contre son principal concurrent, au risque du discrédit explicitement condamné par la loi (Cf. supra, alinéa 2 de l'article L121-12 du Code de la consommation). On aurait donc pu s'attendre à ce que l'attention des parties et du juge portât principalement sur ce point. Or si l'ordonnance de référé évoque effectivement ce grief, c'est non seulement pour le rejeter d'un bref argument le spot a été diffusé pendant la fête d'Halloween, et relève donc davantage de l'humour ${ }^{32}$ que du dénigrement ${ }^{33}$ - mais surtout pour l'abandonner au profit d'autres motifs, qui concernent essentiellement les aspects économico-cognitifs du message. Notons que ces autres aspects sont spontanément évoqués par la partie demanderesse, qui se garde donc bien de faire du dénigrement son seul argument, avant même l'intervention du juge. D'où l'exemplarité

30. Sans nous interdire de faire référence à titre secondaire et complémentaire aux autres affaires ayant opposé les deux opérateurs. L'attention prioritaire portée à l'affaire du vampire tient à la richesse du cas (deux jugements successifs) et à sa convocation dans les autres cas sous forme de jurisprudence.

31. C'est la thèse de France Telecom, selon qui, face à cette publicité, «Le spectateur est nécessairement conduit à assimiler France Télécom à un vampire, de surcroît moribond »; l'image de l'opérateur historique serait celle «d'une entreprise du passé, qui suce le sang de ses clients». Face à cette interprétation Télé 2 objecte que «le vampire ne représente pas France Télécom, mais bien le consommateur qui, s’avisant de vouloir téléphoner de jour, est victime, tel un vampire, de la destruction par la lumière ». Entre ces deux lectures, le juge retiendra celle de France Telecom, mais sans pour autant conclure à son caractère dénigrant : «L'imagerie présentée (décors poussiéreux, cercueil, main de sorcière, flaques rouges...) associe manifestement l'image du vampire à FRANCE TELECOM. Le caractère manifestement excessif de cette comparaison ne dépasse toutefois pas les limites de l'acceptable car il faut tenir compte du fait que la campagne s'est déroulée dans les jours qui précédent Halloween, élément qui en relativise le caractère dénigrant et relève plus de l'humour. »

32. L'humour est sans doute au droit d'aujourd'hui ce que le fou fut au roi de jadis — une façon de rendre « fonctionnelle » une certaine distance irréductible à l'autorité - mais il est important de noter que dans le cas du droit contemporain cette fonctionnalité n'est lisible qu' a posteriori, tant il semble hasardeux de cerner à l'avance ce qui est capable de faire rire un juge.

33. On découvre ici un bel exemple de l'imprévisibilité qui se joue à l'interface de la connaissance ordinaire et préalable du droit et de la prononciation experte et ultérieure des décisions de justice. Pour une sociologie de l'imprévisible, $C f$. Grossetti, 2004. 
paradoxale du cas : si une publicité comparative qui comprend une part non négligeable de fantasmagorie convoque surtout les catégories du calcul et de l'économie, alors on devine à quel point ces mêmes catégories ont des chances de peser dans les formes de comparaison publicitaire pour lesquelles la dimension symbolique passe largement au second plan.

Le jugement qu'émet le juge du Tribunal de commerce chargé d'examiner une affaire de publicité comparative procède de la plainte de l'un des protagonistes, et suit l'exposé des «moyens des parties», c'est-à-dire des argumentaires des plaignants. Ces interventions préalables des parties appellent deux remarques, l'une sur la distribution des compétences juridiques entre les acteurs; l'autre sur le calcul auquel chacun d'entre eux se livre dans l'exercice de ces compétences.

Il existe un différentiel d'expertise évident entre le quidam ordinaire qui lit une affiche et les avocats qui s'en saisissent devant les tribunaux. Tandis que les connaissances juridiques du simple justiciable sont purement hypothétiques (voire fictives, si l'on se réfère à l'adage «nul n'est censé ignorer la loi » évoqué supra), celles des avocats, au contraire, sont réelles et professionnelles: les avocats mobilisent une connaissance approfondie du droit qui se manifeste lorsqu'ils participent à l'élaboration des formes publicitaires, aident à l'évaluation des risques encourus, concourent éventuellement à l'engagement d'une action en justice et, quand cela se produit, lorsque ils fournissent ensuite des arguments aux parties - ces arguments constituant même une matière essentielle pour l'argumentation propre du juge. Par exemple, dans l'affaire du vampire, outre le grief de dénigrement que nous avons déjà évoqué (mais qui n'intervient qu'en troisième position dans l'exposé des moyens !), la société France Telecom (via ses conseils) reproche aussi à son concurrent de procéder à une comparaison inexacte et incomplète des tarifs des deux parties en faisant explicitement référence aux «dispositions applicables en matière de publicité comparative qui font obligation à l'annonceur de décrire complètement les prestations comparées ». Or, c'est aussi en s'appuyant sur les mêmes règles de droit que Télé 2 et ses conseils répondent à cette mise en cause : ils rétorquent que les spots en question ont bien pour contenu de comparer des tarifs précisément indiqués et correspondant à des prestations identiques (la minute de communication hors zone locale dans la journée en semaine), sans oublier de préciser que «le cadre législatif et réglementaire de la publicité comparative fait seulement obligation de comparer des biens ou services effectivement substituables, ce qui est le cas, et pas de comparer l'ensemble des prestations des deux fournisseurs sur lesquels porte la comparaison ».

Cet exemple nous montre que les parties et leurs conseils font preuve d'une égale conviction, appuyée sur une connaissance du droit a priori aussi solide de part et d'autre, et qui débouche sur des argumentaires symétriques visant paradoxalement à fonder un grief (France Telecom) ou au contraire à le récuser (Télé2) en référence aux mêmes règles de droit. Mais dans ces conditions qui, à part le juge, saurait discriminer de façon définitive entre ces arguments à la fois très proches dans la forme et radicalement opposés quant au fond ? En fait, l'examen des litiges invite à ne pas confondre connaissance du droit et préscience du jugement ; comme le dit si bien Lucien Karpik: « La crainte que la justice inspire ne tient pas seulement aux sanctions qu'elle délivre mais aussi et paradoxalement, à l'énigme qu'elle incarne et qui est inséparable de l'incertitude sur le jugement. Un dicton judiciaire l'exprime nettement: on a toujours une chance de gagner le plus mauvais procès et, par réciprocité, on court toujours un risque de perdre la meilleure cause » (Karpik, 1997, p. 104). Or cette 
incertitude sur l'orientation des jugements que Lucien Karpik relève en toute généralité nous semble particulièrement exacerbée en matière de publicité comparative, pour deux raisons. D'une part, le grand nombre de restrictions juridiques associées à cette pratique multiplie les prises pour l'engagement de litiges (Spink \& Petty, 1998), la multiplicité des arguments possibles, et donc la possibilité d'obtenir des conclusions divergentes, d'autant plus difficiles à anticiper qu'elles sont plus nombreuses. D'autre part et surtout, le droit qui régit la publicité comparative sédimente des hésitations qui le rendent intrinsèquement ambivalent: comme l'ont noté les juristes, il semble en effet pour le moins contradictoire d'édicter des règles visant à autoriser cette pratique tout en interdisant qu'elle s'exprime sur le registre du parasitisme, de la concurrence déloyale et du dénigrement, alors que «la publicité comparative relève [pourtant] par définition et par nature du parasitisme », que « les publicités [de ce type] sont de façon inhérente déloyales, compte tenu de leur contenu sélectif (voire déformant) et de leur nature biaisée », et que «le dénigrement est certainement une composante à part entière du medium qui, après tout, est connu dans le langage courant comme "knocking copy" [contrefaçon brutale] » (ibid., p. 863). Ces contradictions rendent l'application du droit qui régit la publicité comparative particulièrement imprévisible: «Les juridictions nationales seront amenées à s'avancer sur une corde raide en matière d'interprétation de cette question [de publicité comparative]. Sachant que les concepts impliqués sont entièrement subjectifs, il est probable que nous ayons à observer de grandes différences d'interprétation et de résultat dans les premiers cas soumis à l'épreuve du droit» (ibid., p. 876). Ainsi, en matière de publicité comparative plus qu'ailleurs peut-être, la justice ne se joue pas uniquement dans la lecture du droit, mais surtout dans la lecture du droit par le juge.

Sans doute, l'incertitude qui prévaut quant à l'orientation des jugements n'exclut pas la mise en œuvre de certaines formes de calcul visant à la réduire, via la mise en balance du rendement économique possible de telle campagne de publicité et du risque juridico-financier encouru. Ce type de calcul en termes de «coûts de juridiction » (calculer la rentabilité économique d'une infraction et/ou le coût d'opportunité de la licéité) est d'ailleurs conforme à ce qu'avance la nouvelle économie institutionnelle lorsqu'elle fait du droit une ressource endogène au calcul économique de la firme ${ }^{34}$. Dans notre cas, l'occurrence d'un calcul intégrant les coûts associés aux décisions de justice se donne explicitement à lire dans les jugements, lorsque la partie demanderesse formule des exigences chiffrées très élevées pour obtenir réparation du préjudice commercial subi (France Telecom demande plus de 1,4 millions d'euros dans l'affaire de 2001, 340500 euros dans celle de 2002, près de 1,8 millions d'euros dans celle de 2003, etc.), mais aussi la réclamation d'astreintes de 15000 euros par infraction constatée (2003) et/ou l'ordonnance d'insertion du jugement sous forme d'encarts dans la presse (pour un montant global de 150000 euros HT en 2002 et 2003), sans oublier l'exigence du remboursement de ses frais de justice conformément à l'article 700 du NCPC (quelques milliers d'euros à chaque fois) et la condamnation aux dépens (quelques dizaines d'euros). Tout se passe comme si ces exigences étaient non seulement une façon de réclamer la correction d'une injustice, mais aussi un moyen d'élever le coût de la publicité comparative, afin de dissuader le challenger de « récidiver » - un objectif que France Telecom formule d'ailleurs très explicitement en 2002,

34. Cf. la proposition d'un marché des droits à polluer chez Coase (1960) ou la prise en compte des coûts inhérents à la mise au point des contrats chez Williamson (1981). 
en requerrant que l'on «interdi[se] à Télé2 de réitérer ce genre d'opération [un concours permettant de gagner un an d'abonnement à l'opérateur public], et d'associer la société FRANCE TELECOM à ses compagnes de promotion ».

Pourtant, il n'est pas certain que les jugements révèlent la totalité des opérations de calcul engagées dans les affaires de publicité comparative. Au contraire, tout se passe comme si chacun des plaignants s'efforçait de masquer le modèle économique qui sous-tend ses prises de position: d'après le jugement de 2001 par exemple, France Telecom aurait évalué son préjudice au coût d'une campagne de publicité, et se serait abstenue de mentionner le nombre de clients (ou la part de marché) qu'une telle campagne lui aurait fait perdre ${ }^{35}$; symétriquement, Télé2 aurait rejeté sur France Telecom la charge de la preuve du préjudice subi (en faisant par exemple observer en 2001 que «la société France Télécom ne prouve aucune perte effective de clientèle, seul préjudice réel qui aurait pu résulter des campagnes incriminées ») et se serait efforcée plus généralement de minimiser l'impact de ses campagnes (par exemple, en 2002, Télé2 dénie toute pertinence aux demandes de réparation de France Télécom non seulement en relevant comme lors de l'affaire précédente que l'entreprise publique «ne prouve pas le préjudice qu'elle allègue », mais aussi en signalant que la campagne incriminée n'a fait l'objet que de quatorze insertions !) Tout se passe comme s'il était également dangereux pour chacune des parties de reconnaître et de mesurer publiquement l'efficacité de la publicité comparative ${ }^{36}$, en raison du risque d'accroître la sanction encourue du point de vue de Télé2 et, symétriquement, du risque de conforter l'efficacité de la méthode et donc d'encourager la « récidive» du challenger du point de vue du plaignant. Face à cette incertitude qui entoure (objectivement et/ou stratégiquement) l'évaluation chiffrée des effets performatifs de la publicité, le juge semble avoir préféré la solution consistant à ne retenir que les sanctions les moins coûteuses : dans les affaires que nous avons examinées, il n'a retenu qu'une fois le paiement de dommages et intérêts en réparation d'un préjudice économique incertain ${ }^{37}$, et s'est contenté généralement d'une part de fixer les sanctions financières aux frais de justice (et aux dépens), d'autre part de prescrire l'insertion du jugement dans divers organes de presse (jugement de 2001) et plus fréquemment sur la page d'accueil du site Internet du challenger (jugements de 2002 et 2003). Au total, on peut se demander si cette «jurisprudence tarifaire » implicite (mais aussi la tendance de France Telecom à ne pas fournir d'éléments de calcul susceptibles de fonder son préjudice) n'a pas participé à la persévérance de Télé 2 dans sa pratique de la publicité comparative, en réduisant peu à peu l'incertitude sur le risque encouru.

35. France Télécom semble avoir attendu le jugement de 2003 pour faire valoir que «son préjudice est substantiel », tout en restant très évasive, puisqu'elle mentionne simplement que «une partie de sa clientèle se détourne de ses offres du fait de la diffusion de messages déloyaux et mensongers »... un point que Télé 2 ne manque pas de relever : «TELE2 soutient que FRANCE TELECOM allègue un préjudice qu'elle ne démontre en rien, et que sa demande d'indemnité devra donc être rejetée ».

36. De façon générale, l'efficacité de la publicité comparative est une question controversée. Pour des études empiriques sur ce point, Cf. Prasad, 1976 ; Goodwin \& Etgar, 1980 ; Demirdjian, 1983, Gotlieb \& Farel, 1991 ; Dianoux, 1999 ; Shao \& al., 2004.

37. Dans le jugement de 2003, le juge justifie explicitement le rejet de la demande de dommages et intérêts en observant que France Telecom «ne fournit aucun élément de preuve selon lequel elle aurait subi un préjudice commercial ou d'image ». Le 17 juin 2004 la cour d'appel de Versailles a condamné Télé2 à payer 43000 euros à France Telecom. 
Le «tour du juge » (moment et savoir-faire) intervient donc au croisement des plaidoiries, des faits économiques et du droit. L'activité du juge consiste-t-elle à corriger des copies traitant de façon différente d'un même sujet, à distribuer des bons et des mauvais points selon que les « élèves » lisent convenablement ou non le droit? Peut-être, mais selon nous là n'est pas le plus important. Nous préférons, pour terminer, laisser de côté le petit jeu anecdotique qui consisterait à vérifier après coup qui a eu gain de cause et sur quel point pour (donner à) gloser sur l'opportunité de la décision (et ses déterminants plus ou moins avouables), et mettre plutôt l'accent sur une création propre du juge à partir de sa lecture du cas et des plaidoiries qui lui sont soumis. Loin de se limiter à la reprise des argumentaires des parties, le juge tente en effet de les dépasser en rapportant le droit à sa conception propre de l'économie. Dans l'affaire du vampire, la façon dont le juge mobilise les conceptions disponibles de l'économie se donne à lire tant au niveau macro qu'au niveau micro.

Au niveau macro, le juge note par exemple que les nouveaux opérateurs « ne peuvent se développer qu'en prenant des parts de marché à l'opérateur historique, le marché des télécommunications fixes ne connaissant pas une croissance comparable à celui des télécommunications mobiles ». Requalifier la concurrence sur le marché du téléphone fixe comme jeu à somme nulle amène le juge à conclure «que lesdits opérateurs sont nécessairement conduits à se comparer à l'opérateur historique, et à faire porter la comparaison sur celles de leurs prestations qui rendent avantageuse pour eux une telle comparaison». Autrement dit, en admettant ainsi qu' un marché peut opposer deux macro acteurs identifiables - un ancien monopole et un challenger privé - et en raisonnant en termes de "parts de marché », non seulement le juge reconnaît et légitime les principes de la concurrence oligopolistique, mais fait de la publicité comparative un attribut quasi «naturel » de ce type de compétition. Cet argumentaire, loin d'être anodin, fera au contraire jurisprudence, puisqu'il sera explicitement puis implicitement repris dans les jugements du 2 juillet 2002 et du 7 mai 2003 (respectivement): on voit ainsi comment les décisions de justice participent, volontairement ou non, à la construction de l'appareillage institutionnel susceptible de permettre l'ouverture effective des services publics à la concurrence.

Cependant, c'est sans doute au niveau micro, en deçà des macro acteurs, que le travail économique du juge est le plus significatif. L'argumentation microéconomique du juge se décline en trois points. D'abord, dans l'ordonnance de référé, le juge déclare qu'il est en pratique impossible de lire le sous-titrage censé préciser les conditions d'application de l'offre (sauf à revoir plusieurs fois le spot), mais observe néanmoins que la seule présence des incrustations signifie que l'offre est soumise à conditions ${ }^{38}$. Ce type de raisonnement, qui consiste à prêter des capacités cognitives aux personnes, se trouve prolongé et précisé dans le jugement suivant, une fois le juge amené à se prononcer sur le fond. Selon lui, le fait que Télé2 limite la comparaison entre son propre tarif (unique) et le tarif «heures pleines » de France Telecom «permet au contraire au spectateur de se poser la question des tarifs dans d'autres tranches horaires » [1]. En outre et d'après le juge, les spots précisent clairement le prix par

38. « Si, en pratique, il n'est pas possible de lire ce qui figure dans le bandeau déroulant et en même temps regarder les images il n'en demeure pas moins que la seule existence du bandeau déroulant signifie que l'offre comporte des conditions. D'autre part la lecture de ce qui est écrit dans ce bandeau déroulant est lisible et un consommateur intéressé peut donc s'y attacher lors d'un passage ultérieur de la publicité.» 
minute, de jour et en semaine pour chaque opérateur. Le juge rappelle à ce propos que la loi n'oblige pas l'annonceur à comparer un ensemble de prestations, mais des prestations clairement identifiées et de même nature, ce qui est le cas ici (tarif unique de Télé 2 vs. tarif «heures pleines» de France Telecom). Par ailleurs, il estime que cette comparaison est pertinente puisqu' elle porte sur le tiers de la durée totale de la semaine [2]. En ce qui concerne l'objectivité de la comparaison, le juge considère en revanche que le message de Télé2 ne permet pas au client de comprendre qu'il s'agit du tarif applicable au-delà du crédit-temps, dont la durée est variable selon les opérateurs. Il fait observer que «les opérateurs de télécommunication mobile savent indiquer que leurs tarifs s'appliquent "dès la première seconde", même dans des publicités brèves ». Selon le Tribunal, l'imprécision dont Télé2 fait preuve sur ce point viole l'obligation de présenter les prestations comparées de manière objective. Il est donc fait interdiction à Télé2 de diffuser les comparaisons incriminées sans les modifier sur ce point [3].

Si l'on examine attentivement chacun des argumentaires présentés, on s'aperçoit que ceuxci mobilisent et définissent une représentation extrêmement fine du sujet économique et des conditions dans lesquelles les activités marchandes doivent s'exercer. En un sens, cette façon de faire n'a rien de nouveau, puisque le juge ne fait que reprendre la fiction juridique du «bon père de famille » à laquelle le droit l'invite à recourir chaque fois qu'il s'agit de trancher des litiges impliquant le point de vue d'un particulier. Mais en matière économique, cette fiction doit encore être précisée, le portrait du «bon père de famille » est ici implicitement redessiné en «bon homo œconomicus». Comment? D'abord, pour estimer les compétences du consommateur ordinaire, le juge se met «à sa place », reprenant ici un geste classique des professionnels du marché (Barrey \& al., 2000). Ce faisant, il prête au consommateur non seulement des compétences perceptuelles (par exemple en termes de vitesse de lecture) mais aussi logiques $(C f$. l'inférence établie entre le défilé d'un bandeau et l'existence de conditions, mais aussi l'inférence seconde, implicitement visée par le juge, d'après laquelle le consommateur est capable de deviner d'une part que ces conditions portent sur les tarifs de France Telecom en heures pleines, et d'autre part que la comparaison pourrait donner d'autres résultats s'il prenait une autre base de comparaison, moins avantageuse pour Télé2). Ces compétences que le juge - et à travers lui le droit — attribuent au consommateur construisent la figure subtile d'un consommateur rationnel, calculateur et doté de discernement (conforme à la vision de l'économie mais aussi à la conception d'un sujet responsable exigée par le droit), mais d'un consommateur néanmoins limité dans ses capacités cognitives (conforme à la vision des théoriciens de la rationalité limitée, mais aussi à la conception d'un sujet faillible, qui nécessite et légitime par conséquent la vigilance et éventuellement la protection de l'institution judiciaire). En d'autres termes, le juge construit ici un «sujet économique de droit» que son argumentaire et son jugement viennent mettre en actes (inscrire dans les textes et dans les comportements).

Cette anthropologisation d'un «sujet économique de droit» se confirme et s'approfondit dans le deuxième argumentaire, qui rend indiscernables les opérations de « jugement» (au sens juridique) et de «calcul» (au sens arithmétique). D'un côté, le juge se réfère aux possibilités d'un calcul «toutes choses égales par ailleurs» telles qu'elles sont inscrites dans le droit (« comparer [...] des prestations clairement identifiées et de même nature »); de l'autre et pour s'y conformer, il rapporte son jugement à une estimation chiffrée de l'adéquation du cas traité 
aux conditions de la loi (le juge calcule que le tarif heures pleines de France Telecom concerne un tiers de la semaine, et en conclut que cette durée est suffisamment conséquente pour justifier une comparaison restreinte à ce tarif) ${ }^{39}$. Le juge traque aussi des défauts de calcul, par exemple lorsqu'il relève une carence d'informations sur le crédit temps pour conclure qu'un tel manque de précision fausse les termes de la comparaison. Ainsi, le calcul se trouve autant exigé que vérifié, de sorte que les promoteurs de la publicité comparative se trouvent ramenés dans un espace de jeu que le juge définit avant tout comme un «espace de calcul » (Callon \& Muniesa, 2004).

Plus généralement, le juge est contraint de mobiliser non seulement la justice, mais aussi sa propre perception de l'état des choses économiques. En effet, ni la réalité ni le droit ne se donnent à lire spontanément ; la compétence du juge porte autant sur l'interprétation du droit — comme on l'admet communément — que sur le décryptage de la réalité — comme nous allons le voir. Les jugements rendus à propos des publicités comparatives opposant Télé2 à France Telecom montrent en effet que le travail du juge consiste autant à interpréter le droit pour traiter le cas empirique qui lui est soumis qu'à interpréter ce cas pour le rendre lisible dans les termes du droit. Pour illustrer ce point, il n'est pas inutile de revenir à l'ordonnance de référé, où le juge estime que comparer France Telecom à un vampire n'entre pas en contradiction avec la lettre de la loi. Tant que l'on ne considère qu'une moitié du travail du juge, qui ferait de lui le gardien exclusif de la lettre de la loi, ce jugement est totalement incompréhensible. En effet, tandis que la loi prohibe explicitement la possibilité de tout dénigrement d'un concurrent - « [La publicité comparative ne peut] Entraîner le discrédit ou le dénigrement des marques, noms commerciaux, autres signes distinctifs, biens, services, activité ou situation d'un concurrent», alinéa 2 de l'article L121-12 du Code de la consommation —, le dictionnaire définit clairement un vampire comme un « mort [...] qui [...] sort de son tombeau pour aller aspirer le sang des vivants » (au sens propre) ou comme un « assassin coupable de crimes mystérieux et sadiques » (au sens figuré). Quelle que soit la définition mobilisée, un vampire reste un vampire, c'est-à-dire une image qui s'apparente bien davantage au dénigrement qu'au compliment! Mais tout s'éclaire lorsque l'on considère l'autre moitié du travail du juge, à savoir celle qui consiste à écrire ensemble le texte et le contexte de la loi : ce qui est dénigrement en soi (un vampire) ne l'est plus lorsqu'on se réfère aux conditions de l'énonciation (Halloween : une fête populaire et/ou commerciale qui consiste à jouer sur la mort).

Cette façon d'instruire la justice en inscrivant le contexte dans la lettre du droit — qui, dans l'ordonnance de référé, s'opère à décharge et sur le registre symbolique — se retrouve dans le jugement rendu sur le fond, cette fois à charge et sur un mode purement économique. En effet, amené à se prononcer sur l'objectivité de la comparaison requise par l'alinéa 3 de l'article L121-8 du Code de la consommation («[Toute publicité comparative n'est licite que si] Elle compare objectivement une ou plusieurs caractéristiques essentielles, pertinentes, vérifiables et

39. L'occurrence de ce calcul propre au juge est d'autant plus remarquable que les parties avaient pourtant tenté de fournir leurs propres argumentaires (plus ou moins) chiffrés : tandis que France Telecom faisait observer que «la plage horaire sur laquelle porte la comparaison n'est pas pertinente, les particuliers, auxquels s'adresse la publicité en cause, téléphonant davantage en soirée et le week-end, pour des raisons évidentes de disponibilité », Télé2 objectait que «46,5\% de la consommation, selon des données publiées par la demanderesse elle-même, interviennent dans la tranche horaire en cause ». 
représentatives de ces biens ou services, dont le prix peut faire partie »), le juge fait remarquer, pour motiver la sanction qu'il prononce sur ce point à l'encontre de Télé2, que « les opérateurs mobiles parviennent à indiquer que leurs tarifs s'appliquent dès la première seconde même dans des publicités brèves... ». Ainsi, loin de mobiliser une définition absolue et décontextualisée de l'objectivité, le juge subordonne plutôt son appréciation à sa propre connaissance économique des pratiques en vigueur dans le secteur des télécommunications. In fine, ce jugement nous montre que ce n'est pas uniquement le droit qui dicte les pratiques de l'économie, mais que c'est aussi l'expérience de ces pratiques qui peut venir nourrir le droit. La prononciation d'un jugement économique avance donc sur une corde raide : d'un côté, le métier de juge consiste uniquement à dire le droit ; de l'autre, parce que les états du monde ne correspondent jamais aux énoncés juridiques, dire le droit amène le juge du Tribunal de commerce à se faire à la fois psychosociologue, économiste et gestionnaire, c'est-à-dire et comme nous l'avons vu, à référer son jugement à une figure possible du sujet économique, à des règles de calcul «toutes choses égales par ailleurs », ainsi qu'à l'évocation de «bonnes pratiques » de l'action concurrentielle. Ainsi, dans les entrailles de la jurisprudence, on découvre que dire le droit c'est aussi dire l'économie, et donc produire une performation «encastrée » de l'économie dans le droit (et réciproquement) : le droit dit et fait ce que l'économie fait et dit, mais aussi ce qu'elle doit dire et faire ${ }^{40}$.

En proposant des modèles cognitifs admissibles, en fixant les conditions nécessaires du calcul, ou en désignant les pratiques gestionnaires acceptables par le droit, l'action du juge contribue bel et bien à faire passer dans la pratique des formes juridiques de l'économie. Sans doute, une telle action économique du droit se heurte à plusieurs limites : la particularité des tribunaux de commerce, dont les juges ne sont pas des magistrats professionnels (Lazega et Mounier, 2003); la singularité et la fragilité de la décision, dont l'argumentation pourra évoluer en appel ou se trouver remise en cause lors d'autres jugements, et dont les attendus ne garantissent en rien qu'elle ne sera pas à nouveau enfreinte par les mêmes ou par d'autres acteurs (comme l'atteste notamment la récurrence des procès concernant un même opérateur) ; la discrétion relative de la décision enfin, dont la visibilité est infiniment moins grande que celle des pratiques examinées. Mais chacune de ces limites renforce paradoxalement les processus en cause plus qu'elle ne vient les minorer.

D'abord, la position particulière des juges accroît leur rôle performatif en matière d'économie : Lise Mounier et Emmanuel Lazega ont bien montré combien la structuration sociale du tribunal de commerce de Paris - soit le même tribunal que celui dont nous avons examiné les jugements ! - contribue à l'exercice d'une gestion particulière des marchés. Par le double lien qu'ils entretiennent avec le monde des affaires (dont ils sont issus, via un processus d'élection complexe visant à assurer une représentation des compétences économiques nécessaires) et avec l'institution judiciaire (pour laquelle ils officient à temps partiel et de façon bénévole), les juges des tribunaux de commerce opèrent une «régulation conjointe » de l'économie, c'est-à-dire une régulation qui croise les contraintes exogènes de la régulation publique avec les impératifs endogènes de la conduite des affaires (Lazega \&

40. On rejoint ici, sur le cas particulier de l'économie, ce qu'observait de façon plus générale Bruno Latour à partir de son ethnographie du Conseil d'État : «sans le reste qui le tient le droit ne serait rien. Reste qu'il tient tout, à sa façon » (Latour, 2002 ; p. 283). 
Mounier, 2003). Notre analyse confirme et prolonge ce résultat, en montrant que ce qui s'observe du côté de la structure relationnelle propre au Tribunal de commerce se retrouve dans les contenus qu'il produit.

Ensuite, la singularité et la fragilité des décisions renforcent paradoxalement leur ancrage dans la double évocation de la lettre du droit et des réalités économiques. En effet, l'accumulation de décisions successives destinées à réajuster continûment les jugements pour accorder au plus près les énoncés juridiques et les données marchandes suscite l'émergence d'un processus d'apprentissage collectif qui s'opère à la fois au sein du droit — c'est tout le sens de la «jurisprudence », qui définit des modes de jugement professionnels partagés — et dans l'univers marchand. À l'examen du contentieux entre Télé2 et France Telecom on s'aperçoit combien les professionnels du marché peuvent se montrer attentifs aux règles de droit : loin de les négliger, de les enfreindre par mégarde, ou de leur opposer leur propre « régulation autonome » (Reynaud, 1988), ils mettent plutôt sciemment les règles officielles «à l'épreuve » (Boltanski \& Thévenot, 1991) ${ }^{41}$, recueillent de la documentation juridique, mobilisent leurs propres juristes et avocats, fourbissent leurs «moyens ». Tandis que les uns tentent de pousser la règle jusqu'à sa limite (par exemple en jouant le jeu périlleux de la publicité comparative), les autres activent la même règle d'autant plus volontiers qu'il s'agit de l'évoquer à l'encontre d'acteurs qui ne font pas partie «de la maison». Et si l'occurrence d'une décision singulière ne garantit en rien son respect ultérieur, elle contribue (au mieux) à border de proche en proche les pratiques possibles - d'une affaire à l'autre, les points incriminés se déplacent et les argumentaires juridiques se multiplient et s'affinent - et parvient (au moins) à mobiliser l'attention des acteurs quant aux enjeux implicites de la double régulation du droit et de l'écono mie qui s'observe en matière de publicité comparative.

Sans doute, les acteurs instruits par les contentieux commerciaux ne constituent qu'un groupe restreint, formé par les plaignants, les avocats, juges et autres juristes, les journalistes spécialisés, les régulateurs, éventuellement les consuméristes, et bien sûr les autres professionnels concernés (publicitaires, autres opérateurs téléphoniques, ${ }^{42}$ etc.). Tout se passe un peu ici comme sur les marchés d'Harrison White (1980), où les acteurs économiques s'observent les uns les autres à travers la scrutation des données marchandes beaucoup plus qu'ils n'observent les consommateurs, sauf qu'ici c'est la scène juridique qui vient remplacer le miroir de l'observation du marché. A priori, la plupart des consommateurs restent à l'écart de ce jeu, tant la publicité dont bénéficient les décisions de justice reste modeste en comparaison de l'exposition dont jouissent les campagnes publicitaires (en dépit des obligations d' « insertion» du jugement dans des journaux sélectionnés ou sur les sites Internet, Cf. par exemple les jugements Télé2 vs. France Telecom des 19 décembre 2001, 23 avril 2003, 7 mai 2003). Si nous avons tous croisé au moins une fois les publicités de Télé2, combien

41. Ce point est d'autant plus frappant dans le secteur de la publicité que la «régulation autonome », par exemple l'action intermédiaire du Bureau de Vérification de la Publicité, emprunte largement au droit : parmi les membres permanents de cette association de professionnels, une bonne partie sont des juristes, d'où un fascinant jeu de chassé-croisé entre les attaches professionnelles des juges, et les attaches juridiques des associations de professionnels (et des annonceurs eux-mêmes).

42. L'exemple de Télé2 a été suivi par d'autres opérateurs, comme par exemple OneTel (http://www.comparatel.fr/news/dnews_id-1124_t-France,Telecom,faire,valoir,de,One,Tel.htm). 
d'entre nous savaient que plusieurs de ces publicités avaient été attaquées en justice, et que la plupart de ces procès avaient au moins partiellement satisfait les plaintes de France Telecom $?^{43}$ Ce différentiel de notoriété est redoutable, dans la mesure où il laisse à penser que les pratiques publiques sont toujours des pratiques licites, participant ipso facto à la généralisation de la publicité comparative dans l'espace marchand. Cette exposition ambiguë contribue pourtant à replacer les consommateurs «oubliés» dans la boucle de l'expérience juridique et marchande concernant la publicité comparative, d'une part en leur montrant, même de façon imparfaite, l'ouverture relative du droit à l'engagement de cette pratique ; d'autre part en leur présentant des modes de saisie des objets économiques (partiellement) conformes à la lettre du droit (accent mis sur des biens et services comparables, prééminence du calcul, examen analytique et rationnel des produits, etc.).

\section{Conclusion}

$\mathrm{Au}$ bout du compte, tandis que certains acteurs ne cessent de se plaindre du caractère envahissant de la régulation publique, l'examen de ce qui se joue autour de la publicité comparative et des affaires qu'elle suscite nous rappelle que cette régulation est aussi très largement désirée, convoquée et «consommée » par les acteurs économiques, et réciproquement (Dumez \& Jeunemaître, 1998). Ainsi, notre examen de la publicité comparative met en scène un double mouvement que l'on aurait tendance à opposer, celui de la pénétration du droit en économie et celui de la pénétration de l'économie en droit (Terré, 1999). L'histoire de la publicité comparative et l'hésitation des acteurs à son encontre montrent la part fondamentale qu'occupe la régulation juridique dans les dynamiques marchandes et dans les stratégies des acteurs économiques ; inversement, la prise en compte par le juge, au moment de dire le droit, des enjeux économiques qui structurent le dossier qui lui est soumis montre aussi à quel point «l'économie tend à occuper une place de plus en plus grande dans le droit» (ibid. 384). Le premier enseignement rejoint l'analyse de Lucien Karpik, qui faisait observer que tout calcul ne peut se comprendre qu'en référence à la «maxime » qui lui donne forme et sens (Karpik, 1998); le second nous apprend que la réciproque est vraie : un jugement prend son sens en vertu du calcul qui le sous-tend. En d'autres termes, si la publicité comparative confirme qu'il y a bien une maxime associée au calcul, elle indique aussi que cette maxime peut ne pas être extérieure et préalable à l'échange mais en constituer éventuellement, parfois, l'objet et le résultat, au terme d'un jeu d'ajustements croisés et tâtonnants entre les ordres économique et juridique qui conduit d'une part les acteurs du marché à appliquer imparfaitement le droit pour faire prospérer leurs affaires, et d'autre part le juge à mobiliser prudemment des conceptions et des données de l'économie pour réaffirmer/réinventer la règle de droit.

Ce jeu qui se met en place autour de la publicité comparative participe à une structuration particulière de l'espace économique, qui consiste à accorder une place croissante aux stratégies de mimétisme et de la différenciation (Pointet, 1997), à la concurrence oligopolistique

43. Un bon indice en la matière nous est donné par certains témoignages exprimés dans des forums de discussion qui semblent montrer que nombre de personnes assimilent occurrence des publicités comparatives et licéité de la pratique. 
(Chamberlin, 1932), à l'expression des choix rationnels et de l'optique consumériste (Mallard, 2000a), etc. ${ }^{44}$, au détriment de rapports économiques moins frontaux, moins calculateurs et moins concentrés. Notons que ce jeu est particulièrement complexe, dans la mesure où la régulation de la publicité comparative oscille entre des impératifs contradictoires: la loi demande en effet que l'affrontement porte sur «des biens ou services répondant aux mêmes besoins ou ayant le même objectif» tout en exigeant que certains acteurs ne tirent pas « indûment profit de la notoriété attachée à une marque »; elle réclame aussi que cette publicité «compare objectivement une ou plusieurs caractéristiques essentielles, pertinentes, vérifiables et représentations de ces biens et services» tout en demandant que cette comparaison n'engendre pas de «confusion entre l'annonceur et un concurrent » et ne présente pas le produit vanté comme «une imitation ou une reproduction d'un bien ou d'un service bénéficiant d'une marque ou d'un nom protégé. » ${ }^{45}$ Ces ambiguïtés du droit — qui semble retirer d'une main ce qu'il accorde de l'autre (Cf. supra, Spink \& Petty, 1998) - se retrouvent dans les hésitations du juge et de l'ensemble des acteurs qui, ne pouvant parvenir à un équilibre stable, s'engagent dans une réévaluation collective et permanente de la règle qu'ils ont à éprouver ensemble, un peu à la manière des jurys analysés en son temps par Garfinkel (1967) et plus récemment par Maynard et Manzo (1993).

Que penser d'un tel processus ? Sans doute, l'autorisation même très limitée de la publicité comparative s'apparente à l'ouverture d'un jeu dangereux, qui produit sans cesse des débordements difficilement contrôlables. Ainsi, après avoir tenté en 2002 de répliquer à la publicité comparative de Télé2 en retournant cette même arme contre son concurrent, le directeur de la communication de France Telecom concluait: "La publicité comparative ne crée pas de valeur. Nous avons ouvert la boîte de Pandore, il faut savoir la refermer » (Girard, 2003). Pourtant, Bruno Latour (2001) nous a récemment rappelé qu'avant de refermer cette fameuse boîte de Pandore, mieux vaut peut-être tenter de l'explorer jusqu'au fond, dans la mesure où l'espoir est censé s'y trouver. Si la publicité comparative tire effectivement les acteurs du marché vers un jeu où chacun risque de se trouver perdant à terme - au gré d'une concurrence destructrice, y compris du point de vue des consommateurs dont les gains tarifaires peuvent avoir pour prix une dégradation symétrique du service - ce jeu circonscrit néanmoins un terrain d'expérimentation du droit et des relations économiques. Précisément: cette fois, l'espoir réside peut-être moins au fond de la boîte que dans l'effort que les acteurs mettent en œuvre pour le dégager. Certes et comme nous l'avons vu, les pratiques des acteurs autour de la publicité comparative sont triplement hésitantes: au cours de l'histoire, le

44. Cette forme de publicité admet en effet la restriction publique du jeu à une série d'acteurs clairement identifiables, et dans lequel on serait chaque fois au stade de la finale et, dans le plus large des cas, des demifinales ( $C f$. le slogan de campagne « Voilà un match gagné d'avance » retenu lors d'une des premières campagnes de Télé2, à l'époque où l'opérateur devait encore gagner sa place de challenger, et étendait donc la comparaison non seulement à France Telecom, mais aussi à 9 Telecom et Cégétel). Il s'agissait de gagner le match de la publicité comparative comme un match de football américain, pourvu que le jeu se déroulât « dans les règles », et même si ces règles sont nombreuses et complexes. Gagner un tel match sans «se mettre à la faute » est non seulement impossible, mais aussi non souhaitable, tant jouer avec les règles fait partie des « sports de contact ».

45. Le problème se pose surtout lorsqu'une marque est associée à un (seul) produit — un cas qui se produit fréquemment dans les publicités comparatives dans la mesure où c'est généralement le leader d'un marché qui est pris à parti. En revanche, en théorie, ce ne sont peut-être pas des impératifs si contradictoires, si l'on dissocie, comme le fait le droit, «marques » d'un côté et « produits » de l'autre. 
législateur a tenté de prendre en compte et de réguler cette pratique, mais il l'a fait en la restreignant fortement ; aujourd'hui, les entreprises se font concurrence, mais il leur reste à choisir si la concurrence consiste ou non à se battre en duel en passant par le droit ; au tribunal, le juge fait comme s'il appliquait le droit, mais il ne peut le faire que prudemment, en s'appuyant aussi à tâtons, avec les autres acteurs, sur des données et des conceptions de l'économie. Pourtant, cette triple hésitation crée un espace de réflexivité, amène chacun à préciser ou à redéfinir ses valeurs, et engage donc une dynamique favorable à l'examen collectif et conscient des politiques et des relations marchandes - un examen que l'on peut espérer porteur d'apprentissages collectifs en matière d'animation sociale des marchés.

\section{Références}

Akerlof, G. A. (1970), 'The market for 'Lemons': Quality Uncertainty and the Market Mechanism," Quarterly Journal of Economics, Vol. 84, 1970, August, pp. 488-500.

Barrey, S. (2004), Le travail marchand dans la grande distribution alimentaire. La définition des relations marchandes, thèse pour le doctorat de sociologie, Toulouse, Université Toulouse II.

Barrey, S., Cochoy, F. \& Dubuisson-Quellier, S. (2000), «Designer, packager et merchandiser : trois professionnels pour une même scène marchande », Sociologie du travail, vol. 42, n 3, pp. 457-482.

Baudrillard, J. (1970), La société de consommation, Paris, Denoël.

Bloor, D. (1982), Socio/logie de la logique ou les limites de l'épistémologie, Paris, Pandore [1976].

Boltanski, L. \& Thévenot, L. (1991), De la justification, Les économies de la grandeur, Paris, Gallimard.

Boltanski, L. (1990), « Sociologie critique ou sociologie de la critique », Politix, n 10-11, pp. 124-134.

Borraz, O. (2004), «Les normes: instruments dépolitisés de l'action publique », in Gouverner par les instruments, Lascoumes, P. \& Le Galès (dir.), Paris, Presses de la FNSP, pp. 123-161.

Boy, L. (dir.) (2004), Le droit de la concurrence : Régulation et/ou contrôle des restrictions à la concurrence, European University Institute, Florence - Published in Italy, June.

Callon, M. \& Muniesa, F. (2004), «Les marchés économiques comme dispositifs collectifs de calcul », Réseaux, vol. $21, \mathrm{n}^{\circ} 122$, pp. 189-233.

Callon, M. (ed.) (1998), The Laws of the Markets, Sociological Review Monographs Series, Oxford, Blackwell.

Canu, R. \& Cochoy, F. (2004), « La loi de 1905 sur la répression des fraudes: un levier décisif pour l'engagement politique des questions de consommation? » Sciences de la société, $\mathrm{n}^{\circ}$ 62, mai, pp. 69-92.

Cas, G. \& Lappare, M. (2001), Droitéconomique : concurrence, distribution, consommation, Paris, Lamy.

Certeau, M. De (1980), L'Invention du quotidien, I, Arts de faire, Paris, UGE 10-18.

Chamberlin E.H. (1932), The theory of monopolistic competition: a reorientation of the Theory of value, Cambridge (Massachusetts), Harvard University Press.

Champaud, C. (2002), «Régulation et droit économique », Revue Internationale de Droit Économique, 1, pp. 2366.

Chessel, M., (1998), La publicité, Naissance d'une profession (1900-1940), Paris, CNRS Editions.

Coase, R.H. (1960), "The problem of social cost", Journal of Law and Economics, Vol. 3, October, pp. 1-44.

Coase, R.H. (1977), “Advertising and free speech", The Journal of Legal Studies, Vol. 6, n 1, pp. 1-34.

Cochoy, F. (1999), Une histoire du marketing, Discipliner l'économie de marché, Paris, La Découverte.

Cochoy, F. (2000), « De 1'“AFNOR” à "NF", ou la progressive marchandisation de la normalisation industrielle », Réseaux, $\mathrm{n}^{\circ} 102$, pp. 65-89.

Cochoy, F. (2002a), Une sociologie du packaging ou l'âne de Buridan face au marché, Paris, PUF.

Cochoy, F. (2002b), «Une petite histoire du client, ou la progressive normalisation du marché et de l'organisation », Sociologie du travail, vol. 44, n 3, juillet-septembre, pp. 357-380.

Cochran, Th. C. (1977), 200 Years of American Business, New York, Basic Books.

Comanor, W.S. \& Wilson, T.A. (1979), "The effect of advertising on competition: A survey", Journal of Economic Literature, Vol. 17, No. 2, pp. 453-476.

Commaille, J. \& Jobert, B. (1999), «La régulation politique: l'émergence d'un nouveau régime de connaissance.», in Les métamorphoses de la régulation politique, Jacques Commaille, and Bruno Jobert (dir.), Paris, LGDJ, pp. 11-33. 
Commons, John R. (1925), “Law and Economics”, The Yale Law Journal, Vol. 34, n 4, pp. 371-382.

Commons, John R. (1931), "Institutional Economics", The American Economic Review, Vol. 21, n 4, pp. 648657.

Demirdjian, Z.S. (1983), "Sales effectiveness of comparative advertising: An experimental field investigation", Journal of Consumer Research, Vol. 10, No.3, pp. 362-364.

Dianoux, Ch. (1999), L'influence des publicités comparatives sur le comportement du consommateur, thèse de doctorat, département Sciences Juridiques, Économiques, Politiques et Gestion, Nancy, IAE de l'Université de Nancy II.

Douriez, B. (2003), «Publicité comparative : qu'a-t-on à y gagner ? Enquête», 60 Millions de consommateurs, $\mathrm{n}^{\circ} 372$, mai, pp. 26-28.

Doyère, J. (1991), «Alors que la loi ne l'autorise pas encore plusieurs journaux insèrent une publicité comparative des centres Leclerc », Le Monde, 19 mars.

Doyle, P. (1968), "Economic Aspects of advertising: A survey", The Economic Journal, Vol.78, No.311, pp. 570-602.

Du Gay, P. (2004), «Self-service: Retail, Shopping and Personhood», Consumption, Markets \& Culture, Vol.7, no. 2, June, pp. 149-163.

Dumez, H. \& Jeunemaittre, A. (1998), 'The unlikely encounter between economics and a market: the case of the cement industry", in The Laws of the Markets, Callon, M. (ed.), Sociological Review Monographs Series, Oxford, Blackwell, pp. 222-243.

Durkheim, É. (1986), Les règles de la méthode sociologique, Paris, Presses Universitaires de France [1937].

Duval, M. (1981), «Les camelots », Ethnologie Française, Vol. 11, n² 2, pp. 62-86.

Ewen, S. (1983), Conscience sous influence. Publicité et genèse de la société de consommation, Paris, Aubier Montaigne.

Farjat, G. (1992), « La notion de droit économique » in Droit et économie, Paris, Sirey, tome 37, pp. 27-62.

Féral, F. (1995), L'État et les fraudes commerciales Essai sur les politiques publiques de contrôle des marchés, Perpignan, Presse Universitaire de Perpignan Collection Études.

Fligstein, N. (1990), The transformation of corporate control, London, Harvard University Press.

Garfinkel, H. (1967), Studies in ethnomethodology, Englewood Cliffs, New Jersey, Prentice-Hall.

Girard, L. (2003), «Les cas de publicité comparative se multiplient en France », Le Monde, 11 février.

Goodwin, S. \& Etgar, M (1980), "An Empirical investigation of comparative advertising: Impact of message appeal, information load, and utility of product class", Journal of Marketing Research, Vol. 17, No.2, pp. 187-202.

Gotlieb, J. B. \& Sarel, D. (1991), «Comparative advertising effectiveness: the role of involvement and source credibility », Journal of Advertising, Vol. 20, No. 1, pp. 38-45.

Grandclément, C. (2004) «Climatiser le marché. Les contributions des marketings de l'ambiance et de l'atmosphère », ethnographiques.org [en ligne] $\mathrm{n}^{\circ} 6$, novembre, http://www.ethnographiques.org/ documents/article/ArGrandclement.html (page consultée le 1er février 2005)

Grossetti, M. (2004), Sociologie de l'imprévisible, Paris, Presses Universitaires de France.

Hennion, A. \& Dubuisson, S. (1996), Design : les objets dans l'usage, Paris, presses de l'École des Mines.

Hennion, A. et Méadel, C. (1988), «Dans les laboratoires du désir : le travail des gens de la publicité », Réseaux, $\mathrm{n}^{\circ} 28$, pp. 9-54.

Kaldor, N. (1950), “The economic aspects of advertising”, The Review of Economic Studies, Vol. 18, No. 1, pp. 127.

Karpik, L. (1989), «L'économie de la qualité », Revue Française de sociologie, vol.30, n², avril-juin, pp.187-210.

Karpik, L. (1997), «L'avancée politique de la justice », Le Débat, n 97, pp. 90-107.

Karpik, L. (1998) «La confiance: réalité ou illusion? Examen critique đune thèse de Williamson», Revue économique, vol. 49, n 4, juillet, pp. 1043-1056.

Karpik, L. (2000), « Le guide rouge Michelin», Sociologie du travail, vol. 42, n 3, pp. 369-389.

Kessous, E. (2004), «Qualité des produits consommés et genèse d'une police du commerce : retour sur les débats de la loi de 1905 sur la répression des fraudes », 3èmes journées normandes de recherche sur la consommation, Sociétés et consommation, Rouen, 11-12 mars.

Kihlstrom, R. \& Riordan, M. (1984), “Advertising as a signal”, The Journal of Political Economy, Vol. 92, No.3, pp. 427-450. 
Kirat, T. (2000), «Action juridique et calcul économique. Regard d'économie du droit», in Kirat, T. \& Serverin, É. (dir.), Le droit dans l'action économique, Paris, CNRS Éditions, 2000, p. 43-56.

Lancaster, K. J. (1975), «Socially optimal product differentiation», American Economic Review, Vol.65, pp. 567-585.

Lapeyronnie, D. (2004), «L'académisme radical ou le monologue sociologique. Avec qui parlent les sociologues ? », Revue française de sociologie, vol. $45, \mathrm{n}^{\circ}$ 4, octobre-décembre, pp. 621-651.

Lascombes, A. \& Legeret C. (1999), La publicité comparative, mémoire de maittrise en sciences économiques, Université des sciences sociales Toulouse I.

Latour, B. (2000), «Du principe de précaution au principe du bon gouvernement : vers de nouvelles règles de la méthode expérimentale », Études, n $393-4$.

Latour, B. (2001), L'espoir de Pandore, Pour une version réaliste de l'activité scientifique, Paris, La Découverte.

Latour, B. (2002), La fabrique du droit, Une ethnographie du Conseil d'État, Paris, La Découverte.

Lazega, E. \& Mounier, L. (2003), «Conventions et structures: le cas du Tribunal de commerce de Paris», Colloque Conventions et institutions, La Défense, Paris, 11-12 décembre.

Le Velly, R (2002), "La notion dencastrement : une sociologie des échanges marchands », Sociologie du Travail, Vol. 44, n 1, pp. 37-53.

Mallard, A. (2000a), «La presse de consommation et le marché. Enquête sur le tiers consumériste », Sociologie du travail, vol. 42, n 3, pp. 391-409.

Mallard, A. (2000b), «L'écriture des normes », Réseaux, n 102, pp. 38-61.

Marshall, A. (1922), Industry and Trade, Macmillan.

Marchand, R. (1986), Advertising, the American Dream, Making Way for Modernity, 1920-1940, Berkeley, University of California Press.

Maynard, D. W. \& Manzo, J. F. (1993), "On the sociology of justice: Theoretical notes from an actual jury deliberation", Sociological theory, Vol. 11, No. 2, pp. 171-193.

Nelson, P. (1970), "Information and consumer Behavior", The Journal of Political Economy, Vol. 78, No.2, pp. 311-329.

Nelson, P. (1974), "Advertising as information”, The journal of Political Economy, Vol. 82, No4, pp. 729-754.

Nelson, P. (1975), “The economic consequences of advertising”, The Journal of Business, Vol. 48, № 2, pp. 213241.

Nouguez, É. (2004), Le prix de la différence. Sociologie économique du médicament générique, mémoire pour le DEA de sociologie, Vatin, F. (dir.), Université Paris X-Nanterre, Paris.

Peyrot, M. (1992), «La publicité comparative et la loi. Les centres Leclerc condamnés pour "dénigrement" des pharmaciens », Le Monde, 10 janvier.

Pinto, L. (1990), «Le consommateur: agent économique et acteur politique », Revue française de sociologie, vol. $31, \mathrm{n}^{\circ} 2$, pp. $179-198$.

Pointet, J.-M. (1997), «Le produit automobile entre différenciation et mimétisme », Les cahiers de recherche GIP Mutations Industrielles, $\mathrm{n}^{\circ} 72,30$ mai.

Polo de Beaulieu, M.-A. (2004), «Enseignes, cris, textes. Les pratiques publicitaires au Moyen Age », Le temps des Médias, $\mathrm{n}^{\circ}$ 2, pp. 8-16.

Pradelle, M. de La (1996), Les vendredis de Carpentras, Paris, Fayard.

Prasad, V. K. (1976), "Communications-Effectiveness of comparative advertising: A laboratory analysis", Journal of Marketing Research, Vol. 13, No. 2, pp. 128-137.

Reynaud, J.-D. (1988), «La régulation dans les organisations : régulation de contrôle et régulation autonome », Revue Française de Sociologie, Vol. 29, n 1 , pp. 5-18.

Sauvageot, A. (1987), Figures de la publicité, figures du monde, Paris, Presses Universitaires de France.

Shao, A.T., Bao, Y \& Gray, E. (2004), "Comparative Advertising Effectiveness: A Cross-Cultural Study", Journal of Current Issues \& Research in Advertising, Fall, Vol. 26, No. 2, pp. 67-80.

Spence, M. (1973), « Job Market Signaling », The Quarterly Journal of Economics, Vol. 87, pp. 355-374.

Spink, P. \& Petty, R. (1998), "Comparative advertising in the European Union", The International and Comparative Law Quarterly, Vol. 47, No.4, pp. 855-876.

Stanziani, A. (2003a), «Produits, normes et dynamiques historiques», Sociologie du travail, vol. $45, \mathrm{n}^{\circ} 2$, pp. 259-266.

Stanziani, A (2003b), «Action économique et contentieux judiciaires. Le cas du plâtrage du vin en France 18511905 », Genèses, pp. 71-90.

Steiner, Ph. (1999), La sociologie économique, Paris, La Découverte. 
Sterk, Stewart E. (1976), "The law of comparative advertising: How much worse is 'better' than 'great", Columbia Law Review, Vol. 76, No. 1, pp. 80-112.

Stigler, G. (1961), "The economics of information," Journal of Political Economy, Vol. 69, No. 3, pp. 213-225.

Strasser, S. (1989), Satisfaction Guaranteed, The Making of the American Mass Market, New York, Pantheon Books.

Swedberg, R. (1997), «Vers une nouvelle sociologie économique: bilan et perspectives », Cahiers internationaux de Sociologie, $\mathrm{n}^{\circ} 103$, pp. 237-263.

Telser, L.G. (1964), “Advertising and competition”, The Journal of Political Economy, Vol. 72, No.6, pp. 537562.

Terré, D. (1999), « Droit et marché », L'année sociologique, Vol. 49, n² 2, pp. 381-406.

Terré, F. \& Frison-Roche, M.-A. (1999), «Sociologie du droit économique — Présentation», L'année sociologique, Vol. 49, $\mathrm{n}^{\circ}$ 2, pp. 281-290.

Tirole, J. (1995), Théorie de l'organisation industrielle, Paris, Economica, deux tomes [1988].

Trigilia, C. (2002), Sociologie économique, Paris, Armand Colin.

Vervaeke, M. (2004), Le design et les immatérialités de l'entreprise, Paris, L'Harmattan.

Weber, M. (1995), Économie et société, tome 1, Les catégories de la sociologie, Paris, Pocket.

White, H. C. (1981), «Where do markets come from? » American Journal of Sociology, Vol. 87, pp. 507-547.

Wilhelm, P. \& Dubarry, M. (2002), «Publicité comparative Le nouveau cadre juridique », Revue Lamy Droit des Affaires, mars.

Williams, R. (1990), « Publicité : le système magique », Réseaux, nº 42, pp. 73-95.

Williamson, O. E. (1981), "The Economics of Organization: The Transaction Cost Approach," The American Journal of Sociology, Vol. 87, November, pp. 548-577. 